\title{
Quelques remarques sur les groupes de Lie algébriques réels
}

\author{
Par Hideya Matsumoto
}

(Reçu le 3 juil., 1964)

\section{Introduction}

Dans un travail [11], I. Satake a associé à une algèbre de Lie semi-simple réelle un diagramme qui la caractérise à un isomorphisme près, que nous appellerons son diagramme de Satake. Le but principal de cet article est d'étudier au point de vue des diagrammes de Satake quelques questions plus ou moins connues sur les groupes algébriques linéaires réels. On traitera la connexion topologique d'un groupe algébrique irréductible, la structure du groupe des automorphismes d'une algèbre de Lie semi-simple et la question de conjugaison des sous-groupes résolubles maximaux et des sous-groupes de Cartan d'un groupe algébrique.

$\mathrm{Au}$ paragraphe 1, nous rappelons d'abord quelques résultats de [11] qui seront essentiels pour la suite de cet article. Nous envisageons ensuite la connexion topologique d'un groupe algébrique réel. On sait que tout groupe algébrique irréductible complexe est topologiquement connexe et que ce n'est pas toujours vrai pour les groupes algébriques irréductibles réels. Nous démontrons que tout groupe algébrique $G$ irréductible réel est le produit semidirect de la composante topologiquement connexe de l'élément neutre dans $G$ par un sous-groupe fini composé d'éléments involutifs.

Le paragraphe 2 est consacré à l'étude des automorphismes d'une algèbre de Lie g semi-simple réelle. Soient $G$ le groupe des automorphismes de $\mathfrak{g}$ et $G^{0}$ celui des automorphismes intérieurs de g. La structure du groupe quotient $G / G^{0}$ a été etudiée par E. Cartan [5] et, après lui, par d'autres auteurs (cf. S. Murakami [10], M. Takeuchi [14]). Nous donnons une méthode permettant de la déterminer à partir du diagramme de Satake de g, et démontrons incidemment, en utilisant la classification des algèbres de Lie simples, que $G$ est le produit semi-direct de $G^{0}$ par un sous-groupe fini $S$, ce qui nous semble avoir été implicitement vérifié depuis E. Cartan [5]. Nous pouvons déterminer explicitement un pareil sous-groupe $S$ pour chaque algèbre de Lie simple réelle.

Le paragraphe 3 est essentiellement relatif à la question de conjugaison 
des sous-algèbres résolubles maximales et des sous-algèbres de Cartan d'une algèbre de Lie g semi-simple réelle. Après avoir traité quelques propriétés des sous-algèbres paraboliques de $g$, nous étudions les sous-algèbres résolubles maximales de $\mathrm{g}$ : nous prouvons que les classes de conjugaison par $G^{0}$ de ces dernières sont en correspondance biunivoque avec celles de sous-algèbres paraboliques de $\mathfrak{g}$ ayant une sous-algèbre semi-simple maximale de la première catégorie. On pourrait déduire ceci immédiatement d’un résultat de G.D. Mostow [9], mais nous nous plaçons à un point de vue un peu différent.

Quant aux sous-algèbres de Cartan, nous ne faisons qu'adapter les résultats de B. Kostant [8] et de M. Sugiura [13] à la théorie des diagrammes de Satake. Par l'intermédiaire des sous-algèbres de Cartan "standard", nous réduisons la question de conjugaison des sous-algèbres de Cartan de $g$ à celle de certains sous-diagrammes du diagramme de Satake de g. Remarquons ici qu'il est naturel, selon nos résultats, de déterminer les classes de conjugaison de sous-algèbres résolubles maximales avant celles de sous-algèbres de Cartan.

Nous donnons à l'appendice quelques résultats obtenus en suivant nos méthodes pour les formes réelles non compactes des algèbres de Lie simples complexes.

Dans cet article, les objets algébriques (espaces vectoriels, algèbres de Lie, groupes algébriques etc.) seront considérés, sauf mention du contraire, sur le corps des réels $R$, et le complexifié d'un objet algébrique $A$ sera désigné par $\tilde{A}$.

Je tiens à exprimer ici ma reconnaissance à $M$. N. Iwahori et à $M . T$. Nagano, qui ont bien voulu diriger mes études. Je les remercie vivement de tous les suggestions et les encouragements qu'ils m'ont donnés au cours de ce travail. Je remercie également M.F. Bruhat, qui a bien voulu me guider de ses conseils pendant son séjour à Tokyo en 1963.

\section{$\S 1$. Préliminaires}

1. Nous allons d'abord rappeler quelques résultats de I. Satake [11], sur lesquels nous nous appuierons dans la suite de ce travail.

Soient $\mathfrak{g}$ une algèbre de Lie semi-simple, $\widetilde{\mathfrak{g}}$ sa complexifiée et $\sigma$ le semiautomorphisme involutif de $\widetilde{\mathfrak{g}}$ défini par g. On sait que $\widetilde{\mathfrak{g}}$ possède une forme réelle compacte $g_{u}$ invariante par $\sigma . \quad \sigma$ commute au semi-automorphisme involutif $\tau$ de $\widetilde{y}$ défini par $g_{u}$, et $g$ se décompose en somme directe de deux sous-espaces $\mathfrak{f}$ et $\mathfrak{p}$, où $\mathfrak{f}=\mathfrak{g} \cap \mathfrak{g}_{u}$ et $\mathfrak{p}=\mathfrak{g} \cap \sqrt{-1} \mathfrak{g}_{n}$. L'automorphisme involutif $\theta=\sigma \tau$ de $\widetilde{\mathfrak{g}}$ laisse invariantes $\mathfrak{g}$ et $\mathfrak{g}_{u}$ respectivement, et la restriction de $\tau$ à $\mathfrak{g}$ s'appelle l'involution de Cartan de $\mathfrak{g}$ associée à la décomposition de $\mathfrak{g}$ ci-

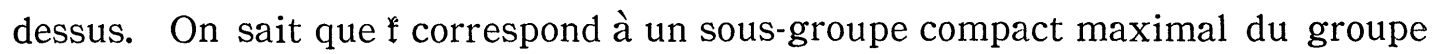
adjoint $G^{0}$ de $g$. 
Soient $\mathfrak{h}-$ une sous-algèbre maximale dans $\mathfrak{p}$ et $\mathfrak{h}$ une sous-algèbre abélienne maximale de $\mathfrak{g}$ contenant $\mathfrak{h}^{-}$. $\mathfrak{h}$ est alors somme directe de $\mathfrak{h}^{+}$et de $\mathfrak{h}^{-}$(où $\mathfrak{h}^{+}=\mathfrak{h} \cap \mathfrak{f}^{\mathfrak{a}}$ et $\mathfrak{h}^{-}=\mathfrak{h} \cap \mathfrak{p}$ ) et donc une sous-algèbre de Cartan de $\mathfrak{g}$. Sa complexifiée $\tilde{\mathfrak{h}}$ est une sous-algèbre de Cartan de $\widetilde{\mathfrak{g}}$ : soit $\Delta$ le système des racines non nulles de $\widetilde{\mathfrak{g}}$ suivant $\tilde{\mathfrak{h}}$. Désignons par $\mathfrak{h}_{0}$ le sous-espace vectoriel sur $R$ composé des vecteurs $h \in \tilde{\mathfrak{h}}$ tels que $\alpha(h)$ soit réel pour toute racine $\alpha$ : on sait que $\mathfrak{h}_{0}$ est somme directe de $\mathfrak{h}$ - et de $\sqrt{-1} \mathfrak{h}^{+}$. La forme de Killing $\langle x, \dot{y}\rangle$ de $\widetilde{g}$ induit sur $\tilde{\mathfrak{h}}$ une forme quadratique non dégénérée qui permet d'identifier $\tilde{\mathfrak{h}}$ et son dual: une racine $\alpha$ sera identifiée avec le vecteur $h_{\alpha} \in \mathfrak{H}_{0}$ tel que l'on ait $\alpha(h)=\left\langle h_{a}, h\right\rangle$ pour tout $h \in \tilde{\mathfrak{h}}$. On voit facilement que $\sigma$ et $\tau$ laissent $\Delta$ invariant et induisent sur $\mathfrak{h}_{0}$ des rotations pour la forme de Killing: on a de plus $\sigma\left|\sqrt{-1} \mathfrak{h}^{+}=-1, \sigma\right| \mathfrak{h}^{-}=1$ et $\tau \mid \mathfrak{h}_{0}=-1$. Nous désignerons désormais par $\sigma_{0}$ la restriction de $\sigma$ à $\mathfrak{h}_{0} . \quad \Delta_{0}=\Delta \cap \sqrt{-1} \mathfrak{h}^{+}$est un système de racines. Nous noterons $W$ [resp. $W_{0}$ ] le groupe de Weyl associé à $\Delta$ [resp. $\left.\Delta_{0}\right]$, c'est-à-dire, le groupe de rotations de $\mathfrak{h}_{0}$ engendré par les symétries $s_{\alpha}$ définies par $s_{\alpha}(h)=h-\frac{2\langle h, \alpha\rangle}{\langle\alpha, \alpha\rangle} \alpha(\alpha \in \Delta)\left[\right.$ resp. $\left.\left(\alpha \in \Delta_{0}\right)\right]$ et désignerons par $W_{\sigma}$ le sousgroupe de $W$ composé des éléments commutant à $\sigma_{0}$. Il est clair que $W_{\sigma}$ contient $W_{0}$. Nous appellerons système $\sigma$-fondamental de $\Delta$ un système foundamental de $\Delta$ tel que, pour toute racine positive $\alpha, \sigma_{0} \alpha$ soit positive ou bien égale à $-\alpha$. On peut ordonner totalement les vecteurs de $\mathfrak{h}_{0}$ par un ordre lexicographique de sorte que le système fondamental de $\Delta$ pour cet ordre soit $\sigma$-fondamental. Soit $\Pi$ un système $\sigma$-fondamental de $\Delta$ et posons $\Pi_{0}=\Pi \cap \Delta_{0}$. On vérifie facilement que $\Pi_{0}$ est un système fondamental de $\Delta_{0}$. En notant $s_{0}$ l'élément involutif dans $W_{0}$ bien déterminé par $s_{0}\left(\Pi_{0}\right)=-\Pi_{0}$, on voit sans peine que $r_{0}=s_{0} \sigma_{0}$ conserve l'ensemble des racines positives et donc $\Pi$ aussi: autrement dit, $r_{0}$ est une rotation particulière de $\mathfrak{h}_{0}$ relative à $I$. De plus on a évidemment $r_{0}^{2}=1$ et $\sigma_{0}=r_{0} s_{0}=s_{0} r_{0}$.

Supposons alors que $\Pi=\left\{\alpha_{1}, \alpha_{2}, \cdots, \alpha_{l-l_{0}}, \alpha_{l-l_{0}+1}, \cdots, \alpha_{l}\right\}, \Pi_{0}=\left\{\alpha_{l-l_{0}+1}, \alpha_{l-l_{0}+2}\right.$, $\left.\ldots, \alpha_{l}\right\}$ où $l$ et $l_{0}$ sont respectivement les rangs de $\Delta$ et de $\Delta_{0}$, et que

$$
\begin{aligned}
& r_{0} \alpha_{i}=\alpha_{i} \quad \text { pour } \quad 1 \leqq i \leqq p_{1}, \\
& \begin{array}{lll}
r_{0} \alpha_{i}=\alpha_{i+p_{2}} & \text { pour } & p_{1}+1 \leqq i \leqq p_{1}+p_{2} \\
\text { et } \quad & \text { pour } & p_{1}+p_{2}+1 \leqq i \leqq l-l_{0}=p_{1}+2 p_{2} . \\
r_{0} \alpha_{i}=\alpha_{i-p_{2}} & \text { poul }
\end{array}
\end{aligned}
$$

Alors, posant $p=p_{1}+p_{2}$ et $\gamma_{i}=\left(\alpha_{i}+\sigma_{0} \alpha_{i}\right) / 2$ pour $1 \leqq i \leqq p$, on voit facilement que $\gamma_{1}, \gamma_{2}, \cdots, \gamma_{p}$ constituent une base de $\mathfrak{h}-$. En outre $\Pi^{-}=\left\{\gamma_{1}, \gamma_{2}, \cdots, \gamma_{p}\right\}$ est un système fondamental d'un système de racines $\Delta^{-}$plongé dans $\mathfrak{h}^{-}([\mathbf{1 1}])$.

Nous pouvons maintenant énoncer le résultat suivant, principalement dû à E. Cartan et à I. Satake (pour la démonstration, voir [11]): 
Théorème 1.1. Désignons par $G^{0}=K^{0} P$ la décomposition de Cartan du groupe adjoint $G^{0}$ de g définie par $\tau \mid \mathrm{g}$.

a. Si une sous-algèbre $\mathfrak{f}^{*}$ de $\mathrm{g}$ correspond à un sous-groupe compact maximal de $G^{0}$, il existe un élément $\xi \in G^{0}$ tel que l'on ait $\xi\left(\mathfrak{l}^{*}\right)=\mathfrak{f}$.

b. Si $\mathfrak{h}^{*}$ est une sous-algèbre de Cartan de g telle que $\mathfrak{h}^{*} \cap \mathfrak{p}$ soit une sousalgèbre maximale dans $\mathfrak{p}$, il existe un élément $\xi \in K^{0}$ tel que l'on ait $\xi\left(\mathfrak{h}^{*}\right)=\mathfrak{h}$.

c. En restreignant à $\mathfrak{h}_{0}$ les opérations du normalisateur $N_{K^{\circ}}(\mathfrak{h})$ de $\mathfrak{h}$ dans $K^{0}$, on obtient un homomorphisme de $N_{K^{\circ}}(\mathfrak{h})$ sur $W_{\sigma}$. de $\Delta$.

d. $W_{\sigma}$ est simplement transitif sur l'ensemble des systèmes $\sigma$-fondamentaux

e. En restreignant à $\mathfrak{h}$ - les opérations de $W_{\sigma}$, on obtient un homomorphisme de $W_{\sigma}$ sur $W^{-}$ayant $W_{0}$ pour noyau, où $W^{-}$désigne le groupe de Weyl associé $\grave{a} \Delta^{-}$.

Le diagramme de Satake de g, désigné par le symbole $(I, \sigma)$, est le diagramme obtenu, à partir du diagramme de Dynkin de $\widetilde{g}$ représentant $\Pi$, en marquant les sommets dans $\Pi_{0}$ et en indiquant par des flèches la restriction à $I-\Pi_{0}$ de la permutation involutive $r_{0}$ (cf. [11], [16]). Nous appellerons $\Pi^{-}$ le systeme fondamental restreint associé à $(\Pi, \sigma)$, et $p=\operatorname{dim} \mathfrak{h}$ - le rang restreint de g ou de $(\Pi, \sigma)$.

D'après le théorème 1.1, le diagramme de Satake de g ne dépend pas du choix de $(\mathfrak{f}, \mathfrak{h}, \Pi)$ : réciproquement, si une algèbre de Lie semi-simple a le diagramme de Satake isomorphe à celui de $g$, c'est isomorphe à $\mathfrak{g}([\mathbf{1}])$.

Soit maintenant $\Pi^{\prime}$ une partie de $\Pi$ telle que $\sigma_{0}$ laisse invariant le sousespace de $\mathfrak{h}_{0}$ sous-tendu par les vecteurs dans $\Pi^{\prime}$. Il existe alors une sousalgèbre semi-simple $g^{\prime}$ de $g$ canoniquement associée à $\Pi^{\prime}$ ([11]) dont le diagramme de Satake est le diagramme $\left(\Pi^{\prime}, \sigma\right)$ obtenu en retirant de $(\Pi, \sigma)$ les sommets n'appartenant pas à $\Pi^{\prime}$ et les traits et flèches qui y aboutissent. Le diagramme $\left(\Pi^{\prime}, \sigma\right)$ sera appelé un sous-diagramme de $(\Pi, \sigma)$.

Nous allons maintenant considérer la catégorie d'une algèbre de Lie semisimple.

Si la restriction de $\theta$ à $\mathfrak{g}_{u}$ est un automorphisme intérieur de $\mathfrak{g}_{u}, \mathfrak{g}$ est dite de la première catégorie: sinon $g$ est dite de la seconde catégorie. Nous dirons que son diagramme de Satake $(\Pi, \sigma)$ est de la première [resp. seconde] catégorie, si $\mathfrak{g}$ est de la première [resp. seconde] catégorie.

Démontrons la proposition suivante, qui nous sera utile plus loin:

PROPOSITION 1.1. a. g est de la première catégorie si et seulement si $\mathrm{g}$ possède une sous-algèbre de Cartan compacte.

b. $(\Pi, \sigma)$ est de la première catégorie si et seulement si $-\sigma_{0}$ appartient à $W$.

DÉmonstration. On rappelle qu'une sous-algèbre de $g$ est dite compacte si elle correspond à un sous-groupe compact de $G^{0}$. On note d'abord que $\mathfrak{f}$ se 
compose de tous les éléments de $g_{u}$ laissés fixes par $\theta$. Comme on le sait, si un automorphisme $\xi$ de $g_{u}$ laisse fixes tous les éléments d'une sous-algèbre de Cartan $\mathfrak{h}_{u}$ de $\mathfrak{g}_{u}$ il existe un èlèment $h \in \mathfrak{h}_{u}$ tel que $\xi=\exp (\operatorname{ad} h)$; et, réciproquement, tout automorphisme intérieur de $g_{u}$ laisse fixes tous les éléments d'au moins une sous-algèbre de Cartan de $g_{u}$ (voir par exemple [12]). La première assertion en résulte immédiatement.

D'autre part $\theta \mid g_{u}$ est intérieur si et seulement si $\theta$ est un automorphisme intérieur de $\tilde{\mathfrak{g}}$, et pour ceci il faut et suffit que $\theta \mid \xi_{0}=-\sigma_{0}$ appartienne à $W$ ([12]) : d'où la deuxième assertion de la proposition 1.1.

2. Nous allons à ce numéro envisager la connexion topologique d'un groupe algébrique irréductible.

Nous rappelons tout d'abord un résultat bien connu sur les groupes algébriques (cf. [6]). Soit $L$ un groupe algébrique d'automorphismes d'un espace vectoriel $V$ de dimension finie. Soient $\mathfrak{l}$ son algèbre de Lie et $\mathfrak{u}$ le plus grand idéal de $\mathfrak{l}$ composé d'éléments nilpotents. $\mathfrak{l}$ est la somme semi-directé de $\mathfrak{l}$ et d'une sous-algèbre réductive $\mathfrak{m}$, et la composante irréductible $L_{1}$ de l'élément neutre dans $L$ est le produit semi-direct de $U$ par $M_{1}$, où $U$ [resp. $M_{1}$ ] est le sous-gropue algébrique irréductible correspondant à $\mathfrak{u}$ [resp. à $\mathfrak{m}]: U$ est le plus grand sous-groupe algébrique unipotent distingué de $L$. Il existe alors un sous-groupe $M$ de $L$ tel que $M$ admette $M_{1}$ comme sous-groupe d'indice fini et tel que $L$ soit le produit semi-direct de $U$ par $M$. De plus, tout sousgroupe algébrique réductif de $L$ est conjugué d'un sous-groupe de $M$.

Nous allons maintenant démontrer le théorème suivant:

THÉORÈme 1.2. Soient $L$ un groupe algébrique irréductible et $L^{0}$ la composante topologiquement connexe de l'élément neutre dans $L$. L possède alors un sous-groupe fini $S$ composé d'éléments involutifs tel que $L$ soit le produit semi-direct de $L^{0}$ par $S$.

DÉmonstration. D'après ce qui précède, pour établir ce théorème, on peut supposer que $L$ soit réductif : car tout groupe algébrique unipotent est topologiquement connexe. Il existe alors une involution de Cartan $\theta$ du groupe $G L(V)$ qui laisse $L$ invariant (voir par exemple [4]) : c'est un automorphisme de $G L(V)$ défini par $\theta(\xi)={ }^{t} \xi^{-1}(\xi \in G L(V))$ où ${ }^{t} \xi$ désigne le transposé de $\xi$ relatif à une forme quadratique définie positive sur $V$. On a donc les décompositions de $L$ et de son algèbre de Lie $\mathfrak{l}$ définies par $\theta: L=K P, \mathfrak{l}=\mathfrak{l}+\mathfrak{p}$. On a alors $L^{0}=K^{0} P$ où $K^{0}$ est la composante connexe de l'élément neutre dans $K$. Soit $\tilde{L}$ le complexifié de $L$ : c'est irréductible et donc topologiquement

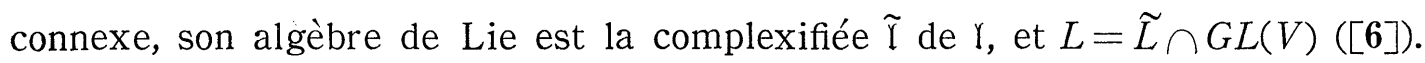
$\theta$ se prolonge en une involution $\tau$ du complexifié $G L(\tilde{V})$ de $G L(V)$ definie par $\tau(\xi)=\left({ }^{t} \bar{\xi}\right)^{-1}(\xi \in G L(\tilde{V})): \tau$ laisse $\widetilde{L}$ invariant. On a donc la décomposition de $\widetilde{L}$ définie par $\tau: \widetilde{L}=L_{u} \exp \left(\sqrt{-1} \mathfrak{Y}_{u}\right)$, où $\mathfrak{\Upsilon}_{u}=\stackrel{*}{+}+\sqrt{-1} \mathfrak{p}$ est l'algèbre de Lie 
du groupe compact connexe $L_{u}$, et on a $K=L_{u} \cap G L(V)$.

Ceci dit, nous allons appliquer le théorème 1.1 à $\mathfrak{l}$ (ou plus précisément à sa sous-algèbre dérivée). Soit $\mathfrak{h}$ une sous-algèbre de Cartan de $\mathfrak{r}$ telle que $\mathfrak{h}-\mathfrak{h} \cap \mathfrak{p}$ soit une sous-algèbre maximale dans $\mathfrak{p}$. Le théorème $1.1 \mathrm{~b}$ entraîne d'abord que $K=K^{0} N_{K}(\mathfrak{h})$ où $N_{K}(\mathfrak{h})$ désigne le normalisateur de $\mathfrak{h}$ dans $K$. Or, quel que soit $\eta \in N_{K}(\mathfrak{h})$, ad $\eta \mid \mathfrak{h}_{0}\left(\mathfrak{h}_{0}=\mathfrak{h}^{-}+\sqrt{-1} \mathfrak{h}^{+}, \mathfrak{h}^{+}=\mathfrak{h} \cap \mathfrak{l}\right)$ laisse $\mathfrak{h}$ - invariant: d'autre part, $\widetilde{L}$ étant connexe, ad $\eta \mid \mathfrak{h}_{0}$ appartient au groupe de Weyl de $\tilde{\mathfrak{l}}$ relatif à $\tilde{\mathfrak{h}}$. On en déduit, par le theorème $1.1 \mathrm{c}$, que $N_{K}(\mathfrak{h})=N_{K^{\circ}}(\mathfrak{h}) Z_{K}(\mathfrak{h}), Z_{K}(\mathfrak{h})$ étant le centralisateur de $\mathfrak{h}$ dans $K$. Il est clair que $Z_{K}(\mathfrak{h})=K \cap Z_{L_{u}}(\mathfrak{h})$, où $Z_{L u}(\mathfrak{h})$ est le centralisateur dans $L_{u}$ de $\mathfrak{h}$, c'est-à-dire de $\mathfrak{h}_{u}=\mathfrak{h}^{+}+\sqrt{-1} \mathfrak{h}$-. Or, puisque $\mathfrak{h}_{u}$ est une sous-algèbre de Cartan de $\mathfrak{I}_{u}$ et puisque $L_{u}$ est compact

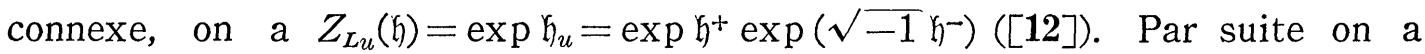
$Z_{K}(\mathfrak{h})=\exp \mathfrak{h}^{+} Q$, où $Q=K \cap \exp (\sqrt{-1} \mathfrak{h}-)$ est l'ensemble des éléments involutifs dans $\exp (\sqrt{-1} \mathfrak{h})$. On a ainsi vu que $K=K^{0} Q$. Soit finalement $S$ un sous-groupe de $Q$ tel que $Q$ soit le produit direct de $Q_{0}=K^{0} \cap Q$ par $S$. Il est alors immédiat que $L$ est le produit semi-direct de $L^{0}$ par $S$, c. q.f. d.

COROllaire. Soit $D$ un tore algébrique $R$-trivial maximal de $L$. On a alors $L=L^{0} D$.

Le corollaire résulte' immédiatement de la démonstration du théorème 1.2.

\section{§ 2. Automorphismes d'une algèbre de Lie semi-simple}

3. Soit g une algèbre de Lie semi-simple. Soient $G$ le groupe algébrique des automorphismes de g, $G_{1}$ [resp. $G^{0}$ ] la composante irréductible [resp. topologiquement connexe] de l'élément neutre dans $G ; G^{0}$ est le groupe adjoint de g.

Nous étudierons dans ce paragraphe la structure du groupe $G$ modulo $G^{0}$ (cf. [5], [10], [14]).

Nous reprenons les notations du $n^{\circ} 1$ et les fixons une fois pour toutes à ce paragraphe.

L'involution de Cartan $\tau \mid g$ définit sur les groupes $G, G_{1}$ et $G^{0}$ leurs décompositions de Cartan respectives:

$$
G=K P, \quad G_{1}=K_{1} P \quad \text { et } \quad G^{0}=K^{0} P .
$$

Soient de plus $G_{u}$ le groupe des automorphismes de $g_{u}$ et $G_{u}^{0}$ le groupe adjoint de $g_{u}$. Les groupes $G$ et $G_{u}$ sont contenus canoniquement dans le groupe des automorphismes de $\widetilde{g}$ que nous désignerons par Aut $(\widetilde{g})$. D'autre part $\tau$ définit sur $\operatorname{Aut}(\widetilde{\mathfrak{g}})$ sa décomposition de Cartan, Aut $(\widetilde{\mathfrak{g}})=G_{u} \exp \left(\sqrt{ }^{-1} \mathfrak{g}_{u}\right)$, et Aut $(\widetilde{\mathfrak{g}})$ n'est autre que le complexifié de $G_{u}$ : celui de $G_{u}^{0}$ ou de $G_{1}$ est la composante irréductible de l'élément neutre dans $\operatorname{Aut}(\widetilde{\mathfrak{g}})$, c'est-à-dire le groupe adjoint 
$\operatorname{Int}(\widetilde{\mathfrak{g}})$ de $\widetilde{\mathfrak{g}}$. Nons avons donc:

$$
\begin{aligned}
& G=\{\xi \mid \xi \in \operatorname{Aut}(\tilde{\mathfrak{g}}), \sigma \xi=\xi \sigma\}, \\
& G_{u}=\{\xi \mid \xi \in \operatorname{Aut}(\tilde{\mathfrak{g}}), \tau \xi=\xi \tau\}, \\
& K=G_{u} \cap G=\left\{\xi \mid \xi \in G_{u}, \theta \xi=\xi \theta\right\}, \\
& K_{1}=G_{u}^{0} \cap G .
\end{aligned}
$$

Nous allons maintenant considérer la décomposition de $\tilde{\mathfrak{g}}$ suivant $\widetilde{\mathfrak{h}}$. On sait qu'il existe une base de $\widetilde{\mathfrak{g}}$ formée de vecteurs $h_{i} \in \mathfrak{h}_{0}(i=1,2, \cdots, l)$ et de vecteurs $e_{\alpha}(\alpha \in \Delta)$ vérifiant les égalités suivantes:

$$
\begin{gathered}
{\left[h, e_{\alpha}\right]=\alpha(h) e_{\alpha} \quad \text { pour } \quad h \in \tilde{\mathfrak{h}},} \\
{\left[e_{\alpha}, e_{-\alpha}\right]=-h_{\alpha},} \\
{\left[e_{\alpha}, e_{\beta}\right]=N_{\alpha, \beta} e_{\alpha+\beta} \quad \text { pour } \quad \alpha, \beta, \alpha+\beta \in \Delta,}
\end{gathered}
$$

les $N_{\alpha, \beta}$ étant des nombres réels non nuls tels que $N_{\alpha, \beta}=N_{-\alpha,-\beta}$,

$$
\tau e_{\alpha}=e_{-\alpha} \quad \text { pour } \quad \alpha \in \Delta .
$$

Une telle base de $\widetilde{g}$ sera appelée une base de Weyl de $\widetilde{g}$ adaptée à sa forme réelle compacte $\mathfrak{g}_{u}$.

Quant au semi-automorphisme $\sigma$, on voit facilement que $\left[h, \sigma e_{\alpha}\right]=\sigma_{0} \alpha(h) \sigma e_{\alpha}$ pour $\alpha \in \Delta$ et $h \in \tilde{\mathfrak{h}}:$ donc $\sigma e_{\alpha}$ est toujours proportionnel à $e_{\sigma_{0} \alpha}$. Nous posons donc:

$$
\sigma e_{\alpha}=\nu_{\alpha} e_{\sigma_{0} \alpha} \quad \text { pour } \quad \alpha \in \Delta .
$$

On vérifie alors les égalités suivantes [11]:

$$
\begin{gathered}
\bar{\nu}_{\alpha} \nu_{\sigma_{0} \alpha}=1, \quad \nu_{\alpha} \nu_{-\alpha}=1, \quad \bar{\nu}_{\alpha}=\nu_{-\alpha}, \quad\left|\nu_{\alpha}\right|=1 \text { pour } \alpha \in \Delta, \\
\nu_{\alpha+\beta} N_{\alpha, \beta}=\nu_{\alpha} \nu_{\beta} N_{\sigma_{0} \alpha, \sigma_{0} \beta} \text { pour } \quad \alpha, \beta, \alpha+\beta \in \Delta, \\
\nu_{\alpha}=1 \quad \text { pour } \quad \alpha \in \Delta_{0} .
\end{gathered}
$$

En vertu de (1.1) et (2.7), il existe un élément $h \in \mathfrak{h}-$ tel que $\nu_{\alpha_{i}}$ $=\exp \left(\sqrt{-1} \alpha_{i}(h)\right)$ pour $\alpha_{i} \in \Pi, 1 \leqq i \leqq p$. En posant $\xi=\exp (\sqrt{-1} \operatorname{ad} h / 2)$ et en remplaçant $e_{\alpha}$ par $\xi e_{\alpha}$, on a, en plus des égalités (6) à (9), $\nu_{\alpha_{i}}=1$ pour $\alpha_{i}$ $(1 \leqq i \leqq p)$ : on en déduit sans peine:

$$
\nu_{\alpha}= \pm 1 \quad \text { pour } \quad \alpha \in \Delta
$$

Remarquons que, si g est normale, c'est-à-dire, si $\mathfrak{h}=\mathfrak{h}^{-}$, on a là de plus $\nu_{\alpha}=1$ pour tout $\alpha \in \Delta$.

Nous fixerons désormais une base de Weyl de $\widetilde{\mathrm{g}}$ adaptée à $\mathfrak{g}_{u}$.

Soit $T_{\sigma}$ le groupe des rotations de $\mathfrak{h}_{0}$ qui laissent $\Delta$ invariant et commutent à $\sigma_{0}$. D'après le théorème 1.1. $\mathrm{d}, T_{\sigma}$ est le produit semi-direct de $W_{\sigma}$ par le sous-groupe Aut $(\Pi, \sigma)$ composé des éléments conservant $\Pi$ : un élément de 
$\operatorname{Aut}(\Pi, \sigma)$ sera appelé automorphisme du diagramme de Satake $(\Pi, \sigma)$. Aut $(\Pi, \sigma)$ se compose des rotations de $\mathfrak{h}_{0}$ qui laissent invariants $\Pi$ et $\Pi_{0}$ respectivement et commutent à $r_{0}$.

LEMmE 2.1. Soit $\xi$ un élément du normalisateur $N_{G u}(\widetilde{\mathfrak{h}})$ de $\tilde{\mathfrak{h}}$ dans $G_{u}$ tel que $\xi \mid \mathfrak{h}_{0}$ appartienne à $T_{\sigma}$. Il existe alors un élément $h \in \mathfrak{h}$ - tel que $\eta=\xi \exp (\sqrt{-1}$ ad $h)$ appartienne au normalisateur $N_{K}(\mathfrak{h})$ de $\mathfrak{h}$ dans $K$.

DÉmonstration. En posant $t=\xi \mid \mathfrak{h}_{0}$, on voit immédiatement que $\xi e_{\alpha}(\alpha \in \Delta)$ est proportionnel à $e_{t \alpha}$ : posons donc $\xi e_{\alpha}=\lambda_{\alpha} e_{t \alpha}$ pour $\alpha \in \Delta$. En vertu de (3) et (5), on vérifie aussitôt:

$$
\lambda_{\alpha} \lambda_{-\alpha}=1, \quad\left|\lambda_{\alpha}\right|=1 \quad \text { pour } \quad \alpha \in \Delta .
$$

En notant que $t$ commute à $\sigma_{0}$, on obtient alors :

$$
\xi^{-1} \sigma \xi \sigma\left(e_{\alpha}\right)=\mu_{\alpha} e_{\alpha} \text { avec } \mu_{\alpha}=\lambda_{\alpha}^{-1} \nu_{t \sigma_{0} \alpha} \bar{\lambda}_{\sigma_{0} \alpha} \bar{\nu}_{\alpha}, \text { pour } \quad \alpha \in \Delta .
$$

D'après (11), on a $\mu_{\alpha}=\bar{\lambda}_{\alpha} \bar{\lambda}_{\sigma_{0} \alpha} \bar{\nu}_{\alpha} \nu_{t \sigma_{0} \alpha}$ et on voit par les égalités (7), (9), (10) que

$$
\begin{array}{lll}
\mu_{\alpha}=\mu_{\sigma_{0} \alpha} & \text { pour } & \alpha \in \Delta, \\
\mu_{\alpha}=1 & \text { pour } & \alpha \in \Delta_{0} .
\end{array}
$$

D'autre part, $\xi^{-1} \sigma \xi \sigma$ étant un automorphisme de $\widetilde{\mathfrak{g}}$, on a

$$
\mu_{\alpha} \mu_{\beta}=\mu_{\alpha+\beta} \quad \text { pour } \quad \alpha, \beta, \alpha+\beta \in \Delta .
$$

On déduit alors sans difficulté des égalités (13) et (14) que l'on a

$$
\mu_{\alpha_{i}}=\mu_{r_{0} \alpha_{i}} \quad \text { pour } \quad \alpha_{i} \in \Pi \text {. }
$$

D'après (13) et (15), il existe un élément $h_{1} \in \mathfrak{h}$ - tel que $\mu_{\alpha_{i}}=\exp \left(\sqrt{-1} \alpha_{i}\left(h_{1}\right)\right)$ pour $\alpha_{i} \in \Pi$, autrement dit tel que $\xi^{-1} \sigma \xi \sigma=\exp \left(\sqrt{-1}\right.$ ad $\left.h_{1}\right)$. Posons finalement $\eta=\xi \exp \left(\sqrt{-1} \operatorname{ad}\left(h_{1} / 2\right)\right)$ : il est facile de vérifier que $\eta$ commute à $\sigma$ et donc appartient au normalisateur $N_{K}(\mathfrak{h})$ de $\mathfrak{h}$ dans $K$. Ceci achève la démonstration du lemme 2.1.

Pour énoncer le théorème principal de ce numéro, nous introduirons quelques sous-groupes finis de $K$. On sait qu'on peut associer à tout $t \in \operatorname{Aut}(\Pi, \sigma)$ un automorphisme $\xi_{t}$ de $g_{u}$ défini par $\xi_{t} e_{\alpha}=e_{t \alpha}, \xi_{t} e_{-\alpha}=e_{-t \alpha}$ pour $\alpha \in \Pi$ : on a $\xi_{t} \mid \mathfrak{h}_{0}=t$. Les éléments $\xi_{t}, t \in \operatorname{Aut}(\Pi, \sigma)$, forment un sous-groupe $A_{\sigma}$ de $G_{u}$, qui est isomorphe à $\operatorname{Aut}(\Pi, \sigma)$. En notant que $A_{\alpha} \exp \left(\sqrt{-1}\right.$ ad $\left.\mathfrak{h}^{-}\right)$est un sous-groupe de $G_{u}$, nous définirons les sous-groupes de $K$ suivants:

$$
\begin{gathered}
Q=G \cap A_{\sigma} \exp \left(\sqrt{-1} \text { ad } \mathfrak{h}^{-}\right), \\
Q_{1}=G_{1} \cap Q, \\
Q_{0}=G^{0} \cap Q .
\end{gathered}
$$

Le groupe $Q$ est contenu dans $N_{K}(\mathfrak{h})$. On a $Q_{1}=G_{1} \cap \exp (\sqrt{-1}$ ad $\mathfrak{h}-)$, car 
$A_{\sigma} \cap \operatorname{Int}(\widetilde{\mathrm{g}})$ se réduit à l'élément neutre (voir par exemple [12]) : par suite $Q_{1}$ se compose des éléments involutifs dans $\exp \left(\sqrt{-1}\right.$ ad $\left.\mathfrak{h}^{-}\right)$, donc est d'ordre $2^{p}$, $p=\operatorname{dim} \mathfrak{h}^{-}$étant le rang restreint de $g$. D'autre part, d'après le lemme $2.1, Q / Q_{1}$ est isomorphe à $\operatorname{Aut}(\Pi, \sigma)$.

Ceci dit, nous allons démontrer le théorème et son corollaire suivants :

THÉORÈme 2.1. On a $G=G^{0} Q$. Le groupe quotient $G / G^{0}$ est isomorphe. à $Q / Q_{0}$.

COROllaire. Le groupe quotient $G / G_{1}$ est isomorphe à Aut $(\Pi, \sigma)$.

DÉmonstration. On déduit facilement du théorème 1.1 que $K=K^{0} N_{K}(\mathfrak{h}, \Pi)$. où $N_{K}(\mathfrak{h}, \Pi)$ désigne le sous-groupe de $N_{K}(\mathfrak{h})$ composé des éléments laissant $\Pi$ invariant. En restreignant à $\mathfrak{h}_{0}$ les opérations de $N_{K}(\mathfrak{h}, \Pi)$, on obtient un homomorphisme $j$ de $N_{K}(\mathfrak{h}, \Pi)$ dans Aut $(\Pi, \sigma)$, son noyau étant $N_{K}(\mathfrak{h}, \Pi) \cap K_{1}$ : d'autre part, comme nous l'avons vu plus haut, $Q$ est contenu dans $N_{K}(\mathfrak{h}, \Pi)$. et l'image de $Q$ par $j$ est identique à $\operatorname{Aut}(\Pi, \sigma)$. On en déduit immédiatement que $N_{K}(\mathfrak{h}, \Pi)=\left(N_{K}(\mathfrak{h}, \Pi) \cap K_{1}\right) Q$, donc que $K=K_{1} Q$. Par ailleurs, la démonstration du théorème 1.2 montre que $K_{1}=K^{0} Q_{1}$. Par suite on a $K=K^{0} Q$, d'où le théorème 2.1. Quant au corollaire, il en résulte immédiatement.

Remarque. $Q$ est une extension du groupe $Q / Q_{0}$ par $Q_{0}$. Cette extension n'est pas toujours inessentielle (cf. $\mathrm{n}^{\circ} 5$, exemple).

4. Nous voulons à ce numéro caractériser les éléments de $Q_{0}$ parmi ceux de $Q_{1}$ et exposer une méthode permettant de déterminer ceux-là explicitement.

Pour $\xi \in G$, nous désignerons par $\mathrm{g}^{\xi}$ la sous-algèbre de $\mathrm{g}$ composée des. éléments fixes de $\xi$ et poserons $\mathfrak{f}^{\xi}=\mathrm{g}^{\xi} \cap \mathfrak{q}$.

Nous allons tout d'abord démontrer la

Proposition 2.1. Supposons que g soit simple et non compacte. Désignons. par $Z$ le centralisateur de dans $G$.

a. L'involution de Cartan $\theta$ est le seul élément involutif non neutre dans $Z$.

b. Si g est de la première catégorie, $Z$ est contenu dans $K^{0}$.

c. Si g est de la seconde catégorie, $Z \cap K_{1}$ se réduit à l'élément neutre.

DÉmonstration. On rappelle que $K$ est le normalisateur de flans $G ; Z$ est donc contenu dans $K$. D'autre part on sait que, $g$ étant simple, $f$ est une sous-algèbre propre maximale dans $g$ : la première assertion en résulte immédiatement.

Supposons que $g$ soit de la première catégorie. $\mathfrak{f}$ contient alors une sousalgèbre de Cartan $\mathfrak{h}_{u}$ de $g_{u}$ (proposition 1.1); d'autre part on sait que le centralisateur de $\mathfrak{h}_{u}$ dans $G_{u}$ est contenu dans $\exp \left(\operatorname{ad} \mathfrak{h}_{u}\right)$. Ceci implique la deuxième assertion.

Supposons ensuite que $Z \cap K_{1}$ renferme un élément $\zeta$ non neutre. Puisque $\mathfrak{f}$ est maximale dans $\mathfrak{g}, \mathrm{g}^{\zeta}$ est égale à $\mathfrak{f}$. est alors identique aussi à la sous- 
algèbre de $g_{u}$ composée des éléments fixes de $\zeta$, et donc contient une sousalgèbre de Cartan de $\mathfrak{g}_{u}$; car $\zeta$ appartient à $G_{u}^{0}$. D'après la proposition 1.1, $\mathfrak{g}$ est alors de la première catégorie. Cela prouve la dernière assertion de la proposition 2.1.

REMARQUE. La proposition 2.1 a implique que le tore $Z^{0}$ est de dimension au plus 1. En fait on sait que $Z$ est fini d'ordre 2 ou bien un tore de dimen$\operatorname{sion} 1([2])$.

Corollaire 1. Pour qu'un $\xi \in K_{1}$ appartienne à $K^{0}$, il faut et il suffit que $\mathfrak{l}^{\mathfrak{s}}$ soit de même rang que $\mathfrak{\text { f }}$.

Corollaire 2. Supposons g simple. Si g est de la première [resp. seconde]

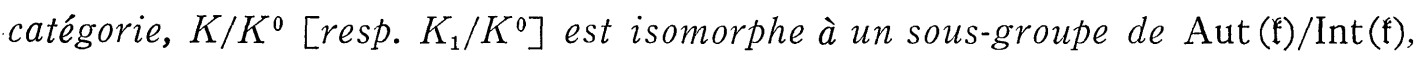
où Aut $(\mathfrak{f})$ désigne le groupe des automorphismes de $\mathfrak{f}$ étant involutifs sur le centre de $\mathfrak{f}$, et Int $(\mathfrak{f})$ désigne la composante connexe de l'élément neutre dans Aut (f).

Les corollaires résultent facilement de la proposition 2.1 .

Nous allons maintenant introduire une sous-algèbre semi-simple normale

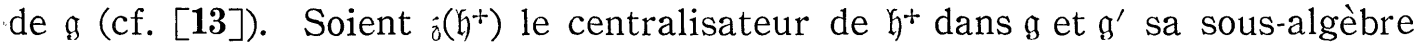
dérivée: soient de plus $\mathfrak{h}^{\prime}$ le sous-espace de $\mathfrak{h}^{-}$-sous-tendu par les éléments dans $\Delta^{\prime}=\Delta \cap \mathfrak{h}^{-}$et $\mathfrak{h}^{\prime \prime}$ le supplémentaire orthogonal de $\mathfrak{h}^{\prime}$ dans $\mathfrak{h}^{-}$. On vérifie facilement que $z\left(\mathfrak{h}^{+}\right)$est la somme direct de $\mathfrak{g}^{\prime}$ et de $\mathfrak{h}^{+}+\mathfrak{h}^{\prime \prime}$, et que $\mathfrak{h}^{\prime}$ est une sous-algèbre de Cartan de $\mathfrak{g}^{\prime}$ : donc $\mathfrak{g}^{\prime}$ est semi-simple normale. D'autre part, $\mathfrak{g}^{\prime}$ étant invariante par $\tau$, on a $\mathfrak{g}^{\prime}=\mathfrak{l}^{\prime}+\mathfrak{p}^{\prime}$ où $\mathfrak{l}^{\prime}=\mathfrak{f} \cap \mathfrak{g}^{\prime}$ et $\mathfrak{p}^{\prime}=\mathfrak{p} \cap \mathfrak{g}^{\prime}$, et $\mathfrak{z}\left(\mathfrak{h}^{+}\right) \cap \mathfrak{f}$ $=\mathfrak{h}^{+}+\mathfrak{H}^{\prime}$.

On peut voir facilement que $\Delta^{\prime}=\Delta \cap \mathfrak{h}$ - est le système de racines de $\mathfrak{g}^{\prime}$ relatif à $\mathfrak{h}^{\prime}$, et que le diagramme de Satake de $\mathfrak{g}^{\prime}$ est isomorphe à un sousdiagramme de celui de la forme réelle normale de $\widetilde{g}$. On a aussi :

$$
\Delta^{\prime}=\left\{\beta \mid \beta \in \Delta,\left\langle\beta, \Pi_{0}\right\rangle=0, r_{0} \beta=\beta\right\} .
$$

Nous définirons les groupes finis $Q_{1}^{\prime}$ et $Q_{0}^{\prime}$ d'automorphismes de $g^{\prime}$ (cf. (16), (17), (18)) :

$$
Q_{1}^{\prime}=\operatorname{Aut}\left(\mathfrak{g}^{\prime}\right) \cap \exp \left(\sqrt{-1} \operatorname{ad}_{\mathfrak{g}}, \mathfrak{h}^{\prime}\right), Q_{0}^{\prime}=\operatorname{Int}\left(\mathfrak{g}^{\prime}\right) \cap Q_{1}^{\prime},
$$

où Aut $\left(\mathfrak{g}^{\prime}\right)$ [resp. Int $\left(\mathfrak{g}^{\prime}\right)$ ] désigne le groupe des automorphismes [resp. des automorphismes [intérieurs] de $\mathfrak{g}^{\prime}$.

Ceci posé, les rapports existant entre $Q_{1}$ et $Q_{1}^{\prime}$ sont exprimés par le lemme suivant :

LEMME 2.2. Tout élément de $Q_{1}$ laisse $\mathrm{g}^{\prime}$ invariante: en restreignant à $\mathrm{g}^{\prime}$ les opérations de $Q_{1}$, on obtient un homomorphisme $j$ de $Q_{1}$ dans $Q_{1}^{\prime}$. En outre, pour qu'un $\omega \in Q_{1}$ appartienne à $Q_{0}$, il faut et il suffit que $j(\omega)$ appartienne à $Q_{0}^{\prime}$.

DÉmONSTRATION. Il est clair que $z\left(\mathfrak{h}^{+}\right)$et $\mathfrak{g}^{\prime}$ sont invariantes par tout 
automorphisme de $\mathfrak{g}$ laissant $\mathfrak{h}^{+}$invariant. En désignant par $j$ la restriction à $\mathfrak{g}^{\prime}$ de tels automorphismes de $\mathfrak{g}$, on a, dans la complexification, $j\left(\exp \left(\sqrt{-1}\right.\right.$ ad $\left.\left.\mathfrak{h}^{-}\right)\right)$ $=j\left(\exp \left(\sqrt{-1} \operatorname{ad} \mathfrak{h}^{\prime}\right) \exp \left(\sqrt{-1}{\operatorname{ad~} \mathfrak{h}^{\prime \prime}}^{\prime}\right)\right)=j\left(\exp \left(\sqrt{-1} \operatorname{ad~h}^{\prime}\right)\right)=\exp \left(\sqrt{-1} \operatorname{ad}_{g^{\prime}} \mathfrak{h}^{\prime}\right):$ d'où la première assertion.

Comme nous l'avons vu plus haut, on a:

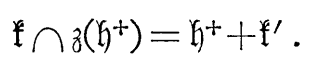

On a donc plus généralement, pour $\omega \in Q_{1}$,

$$
\mathfrak{l}^{\omega} \cap \mathfrak{z}^{\left(\mathfrak{h}^{+}\right)}=\mathfrak{h}^{+}+\mathfrak{l}^{\prime j(\omega)},
$$

en posant $\mathfrak{f}^{\prime j(\omega)}=\mathfrak{f}^{(\omega)} \cap \mathfrak{k}^{\prime}$. D'autre part on sait que $\mathfrak{f} \cap z^{\prime}\left(\mathfrak{h}^{+}\right)$[resp. $\left.\mathfrak{f}^{\omega} \cap z^{\prime}\left(\mathfrak{h}^{+}\right)\right]$est de même rang que $\mathfrak{f}\left[\right.$ resp. $\left.\mathfrak{l}^{\omega}\right]$. En vertu de $(21)$ et $(22)$, it en résulte que $\mathfrak{f}^{\omega}$ est de même rang que $\mathfrak{f}^{\mathfrak{y}}$ si et seulement si $\mathfrak{f}^{\prime j(\omega)}$ est de même rang que $\mathfrak{f}^{\prime}$. Par le corollaire 1 à la proposition 2.1, ceci démontre la deuxième assertion du lemme 2.2 .

Nous pouvons maintenant dessiner une méthode permettant de déterminer explicitement les éléments de $Q_{0}$. Rappelons tout d'abord que le système fondamental restreint $\Pi^{-}$associé à $(\Pi, \sigma)$ est une base de $\mathfrak{h}^{-}$:

$$
\Pi^{-}=\left\{\gamma_{1}, \gamma_{2}, \cdots, \gamma_{p}\right\} \text {. }
$$

Soit $\left\{\varepsilon_{1}, \varepsilon_{2}, \cdots, \varepsilon_{p}\right\}$ la base de $\mathfrak{h}^{-}$duale à $\Pi^{-}$:

$$
\left\langle\varepsilon_{i}, \gamma_{j}\right\rangle=\delta_{i j} \quad \text { pour } \quad 1 \leqq i, j \leqq p .
$$

Alors, en posant

$$
\omega_{i}=\exp \left(\sqrt{-1} \operatorname{ad}\left(\pi \varepsilon_{i}\right)\right) \quad \text { pour } \quad 1 \leqq i \leqq p,
$$

on voit facilement que $\omega_{1}, \omega_{2}, \cdots, \omega_{p}$ constituent une base canonique du groupe $Q_{1}$ fini abélien.

$1^{\circ}$ Plaçons-nous d'abord dans le cas où g est normale. Dans ce cas, pour $\omega \in Q_{1}, \mathrm{~g}^{\omega}$ est aussi réductive normale et de même rang que g. Pour $\omega=\omega_{i}$ $(i=1,2, \cdots, p)$ et pour d'autres $\omega \in Q_{1}$, en déterminant un système fondamental du système de racines $\Delta^{\omega}$ de $\mathfrak{g}^{\omega}$ et en comparant ensuite le rang de $\mathfrak{f}^{\omega}$ avec celui de f́, on examine, par le corollaire 1 à la proposition 2.1, si $\omega$ appartient à $Q_{0}$. On peut ainsi déterminer les éléments de $Q_{0}$.

$2^{\circ}$ Dans le cas général, on détermine d'abord le système fondamental $\Pi^{\prime}$ de $\Delta^{\prime}$ à partir du diagramme de Satake $(\Pi, \sigma)$ de g (voir (2.19)] et puis l'homomorphisme $j: Q_{1} \rightarrow Q_{1}^{\prime}$. D'après le lemme 2.2, on peut alors déterminer les éléments de $Q_{0}$ en utilisant le résultat déjà obtenu dans le cas normal.

REMARQUE. a. Pour effectuer ces procédés, il nous faut connaître, pour toute algèbre de Lie simple $g$ normale, le rang de $\mathfrak{f}$ et la racine dominante en forme de combinaison linéaire des racines simples.

b. Le corollaire 2 à la proposition 2.1 permet d'obtenir une borne supérieure 
pour l'ordre de $Q / Q_{0}$ ou de $Q_{1} / Q_{0}$ en voyant le type de $\mathfrak{f}$ (cf. aussi $\mathrm{n}^{\circ} 7$ ).

5. Rappelons brièvement quelques résultats bien connus sur les algèbres de Lie simples complexes et réelles.

On sait que la famille des algèbres de Lie simples complexes (à un isomorphisme près) se compose de quatre classes d'algèbres simples classiques, désignées par les symboles $\left(\mathrm{A}_{n}\right)(n \geqq 1),\left(\mathrm{B}_{n}\right)(n \geqq 2)$, $\left(\mathrm{C}_{n}\right)(n \geqq 3)$ et $\left(\mathrm{D}_{n}\right)(n \geqq 4)$, et en outre de cinq algèbres simples exceptionnelles, désignées par $\left(\mathrm{E}_{n}\right)$ $(n=6,7,8),\left(\mathrm{F}_{4}\right)$ et $\left(\mathrm{G}_{2}\right)$.

Soient $\tilde{\mathfrak{g}}$ une algèbre de Lie simple complexe et $\tilde{\mathfrak{h}}$ une sous-algèbre de Cartan de $\tilde{\mathfrak{g}}$. Désignons par $T$ le groupe des transformations linéaires de $\widetilde{\mathfrak{h}}$ conservant le système de racines $\Delta$ de $\tilde{\mathfrak{g}}$ relatif à $\tilde{\mathfrak{h}}$, et par $W$ le groupe de Weyl associé à $\Delta$. On sait que le groupe quotient Aut $(\widetilde{\mathrm{g}}) / \operatorname{Int}(\widetilde{\mathrm{g}})$ est isomorphe à $T / W$ et aussi au groupe des automorphismes du diagramme de Dynkin de $\tilde{\mathrm{g}}$ : réellement il est isomorphe à :

$$
\left\{\begin{array}{l}
S_{3}, \text { si } \widetilde{g} \text { est de type }\left(\mathrm{D}_{4}\right), \\
Z_{2} \text {, si } \widetilde{g} \text { est de l'un des types }\left(\mathrm{A}_{n}\right)(n \geqq 2),\left(\mathrm{D}_{n}\right)(n \geqq 5) \text { et }\left(\mathrm{E}_{6}\right), \\
\{1\}, \text { sinon; }
\end{array}\right.
$$

où $S_{3}$ désigne le groupe symétrique de degré 3 , et $Z_{2}$ le groupe cyclique d'ordre 2.

D'autre part la transformation scalaire -1 appartient toujours à $T$ : on sait qu'elle n'appartient pas à $W$ si et seulement si $\tilde{\mathfrak{g}}$ est de l'un des types $\left(\mathrm{A}_{n}\right)(n \geqq 2),\left(\mathrm{D}_{2 m+1}\right)(m \geqq 2)$ et $\left(\mathrm{E}_{6}\right)$. Ainsi, si Aut $(\widetilde{\mathfrak{g}})$ n'est pas identique à Int $(\tilde{\mathfrak{g}})$ et si -1 appartient à $W, \widetilde{\mathfrak{g}}$ est de l'un des types $\left(\mathrm{D}_{2 m}\right)(m \geqq 2)$.

Soit maintenant $g$ une algèbre de Lie simple: $g$ est ou bien une forme réelle d'une algèbre de Lie simple complexe ou bien l'algèbre de Lie obtenue par la restriction scalaire d'une algèbre de Lie simple complexe. On sait que toute algèbre de Lie simple complexe possède une et une seule forme réelle normale à un isomorphisme près.

En vue d'une utilisation ultérieure, nous allons déterminer, suivant la méthode exposée au numéro précédent, le groupe quotient $Q / Q_{0}$ pour une algèbre de Lie simple normale $g_{0}$ de type $\left(D_{n}\right)(n \geqq 4)$. On peut supposer, en vertu de (10), que $A_{\sigma}$ soit contenu dans $K$, donc que $Q$ soit le produit semidirect de $Q_{1}$ par $A_{\sigma}$ (voir (16)). Prenons le diagramme de Satake $(\Pi, \sigma)$ de $g_{0}$ :

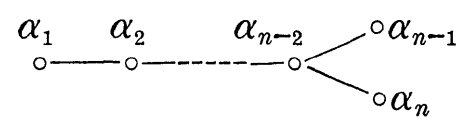

et la base canonique de $Q_{1},\left\{\omega_{1}, \omega_{2}, \cdots, \omega_{n}\right\}$, associée à $\Pi$ par (24), (25). Rappelons ici que: i) si g est simple normale de type $\left(\mathrm{A}_{n}\right), \mathfrak{f}$ est de rang $\left[\frac{n+1}{2}\right]$; 
ii) si g est simple normale de type $\left(D_{n}\right)$, l est de rang $2\left[\begin{array}{c}n \\ 2\end{array}\right]$ (cf. $n^{\circ} 10$ ).

Ceci dit, jetant un regard sur les racines positives, on vérifie que $\mathfrak{g}_{0}^{\omega}$ est de type:

$$
\begin{array}{lll}
\left(\mathrm{D}_{n-i}\right)+\left(\mathrm{D}_{i}\right) & \text { pour } & \omega_{i}(1 \leqq i \leqq n-2), \\
\left(\mathrm{A}_{n-1}\right)+\left(\mathrm{D}_{1}\right) & \text { pour } & \omega_{j}(j=n-1, n), \\
\left(\mathrm{D}_{n-i+1}\right)+\left(\mathrm{D}_{i-1}\right) & \text { pour } & \omega_{1} \omega_{i}(2 \leqq i \leqq n-2), \\
\left(\mathrm{D}_{n-1}\right)+\left(\mathrm{D}_{1}\right) & \text { pour } & \omega_{n-1} \omega_{n} \\
\left(\mathrm{D}_{n-2}\right)+\left(\mathrm{D}_{2}\right) & \text { pour } & \omega_{1} \omega_{n-1} \omega_{n}
\end{array}
$$

où $\left(D_{1}\right)$ désigne l'algèbre de Lie d'un tore algébrique de dimension $1,\left(D_{2}\right)$ et $\left(D_{3}\right)$ signifient $\left(A_{1}\right)+\left(A_{1}\right)$ et $\left(A_{3}\right)$ respectivement. Faisant usage de ce qu'on vient de rappeler, on en déduit les relations modulo $Q_{0}$ suivantes:

pour $n$ impair,

pour $n$ pair,

$$
\begin{aligned}
& \omega_{1} \equiv \omega_{2} \equiv \cdots \equiv \omega_{n-2} \equiv 1, \\
& \omega_{n-1} \equiv \omega_{n} \equiv 1 ;
\end{aligned}
$$

$$
\begin{aligned}
& \omega_{2} \equiv \omega_{4} \equiv \cdots \equiv \omega_{n-2} \equiv 1, \\
& \omega_{n-1} \neq 1, \omega_{n} \equiv 1, \omega_{1} \equiv \omega_{3} \equiv \cdots \equiv \omega_{n-3} \equiv \omega_{n-1} \omega_{n} \equiv 1 .
\end{aligned}
$$

On en conclut d'abord que $Q / Q_{0}$ est isomorphe à $Z_{2} \times Z_{2}$ pour $n$ impair. Pour $n$ pair, en posant $S_{1}=\left\{1, \omega_{3} \omega_{4}, \omega_{4} \omega_{1}, \omega_{1} \omega_{3}\right\}(n=4)$ ou $S_{1}=\left\{1, \omega_{n-1}, \omega_{n}, \omega_{n-1} \omega_{n}\right\}$ ( $n \geqq 6$ ), on voit facilement que $Q_{1}$ est le produit direct de $Q_{0}$ par $S_{1}$ et que $S_{1}$ est invariant par $A_{\sigma}$. $Q$ est donc le produit semi-direct de $Q_{0} \operatorname{par} S=S_{1} A_{\sigma}$ : par suite $G$ est aussi le produit semi-direct de $G^{0}$ par $S$. On en déduit que $Q / Q_{0}$ est isomorphe à $S_{4}(n=4)$ ou à $D_{4}(n \geqq 6) ; S_{4}$ désigne ici le groupe symétrique de degré 4 , et $D_{4}$ le groupe diédrique d'ordre 8.

6. A ce numéro, à l'aide de quelques vérifications utilisant la classification des algèbres de Lie simples, nous allons démontrer que $G$ est le produit semi-direct de $G^{0}$ par un sous-groupe fini.

Vérifions d'abord la proposition suivante:

Proposition 2.2. Supposons que g soit une forme réelle non compacte d'une algèbre de Lie simple complexe et que $G$ ne soit pas identique à $G_{1}, I l$ existe alors un automorphisme involutif $\xi$ de g tel que i) $G=G_{1}\{1, \xi\}$, ii) $\xi \in N_{K}(\mathfrak{h}), \xi \mid \mathfrak{h}-=-1$, sauf dans le cas où g soit isomorphe à l'une des formes réelles normales de types $\left(\mathrm{D}_{2 m}\right)(m \geqq 2)$.

DÉmonstration. D'après ce qu'on a rappelé au numéro précédent, l'hypothèse faite entraîne que $\tilde{\mathfrak{g}}$ est de l'un des types $\left(\mathrm{A}_{n}\right)(n \geqq 2),\left(\mathrm{D}_{n}\right)(n \geqq 4)$ et $\left(\mathrm{E}_{6}\right)$. Nous distinguons quelques cas:

$1^{\circ}$. Supposons que g soit de la seconde catégorie. On voit alors immédiate- 
ment que Aut $(\Pi, \sigma)$ est d'ordre 2 et que l'involution de Cartan $\theta$ vérifie les propriétés requises pour $\xi$.

$2^{\circ}$. Supposons que $\tilde{\mathfrak{g}}$ soit de l'un des types $\left(\mathrm{A}_{n}\right)(n \geqq 2),\left(\mathrm{D}_{2 m+1}\right)(m \geqq 2)$, $\left(\mathrm{E}_{6}\right)$. Soit alors $\xi$ l'automorphisme de $\tilde{g}$ bien défini par $\xi e_{\alpha}=e_{-\alpha}(\alpha \in \Delta)$ par rapport à la base de Weyl vérifiant encore (2.10): c'est un automorphisme involutif de $g$ qui n'appartient pas à $G_{1}$. Ceci entraîne immédiatement notre assertion dans ce cas.

$3^{\circ}$. Supposons que $\tilde{\mathfrak{g}}$ soit de l'un des types $\left(D_{2 m}\right)(m \geqq 2)$ et que $\mathfrak{g}$ ne soit pas normale. Un coup d'œil sur les diagrammes de Satake des algèbres de Lie de cette sorte montre que Aut $(\Pi, \sigma)$ est d'ordre 2 (s'il n'est pas neutre) et que son élément non neutre $t$ induit sur $\mathfrak{h}$ - l'application identique (cf. appendice). Soit maintenant $\eta$ l'automorphisme involutif de $\widetilde{\mathfrak{g}}$ défini par

$$
\eta e_{\alpha}=e_{-t \alpha}, \quad \eta e_{-\alpha}=e_{t \alpha} \quad \text { pour } \quad \alpha \in I I .
$$

Par le lemme 2.1, il existe un automorphisme $\xi$ de $g$ de la forme $\xi=$ $\eta(\sqrt{-1}$ ad $h), h \in \mathfrak{h}^{-}:$l'égalité $\eta \mid \mathfrak{h}^{-}=-1$ entraîne que $\xi$ est aussi involutif. - $t$ n'appartenant pas à $W$, on a $G=G_{1}\{1, \xi\}$. Ceci achève la démonstration de la proposition 2.2 .

Nous sommes maintenant en état de prouver le théorème suivant:

THÉORÈme 2.2. G possède un sous-groupe fini S tel que

i) $G$ soit le produit semi-direct de $G^{0}$ par $S$,

ii) $S$ soit le produit semi-direct de $G_{1} \cap S$ par un sous-groupe $E$.

DÉmonstration. Pour établir ce théorème, on peut supposer sans nuire la généralité que $g$ soit simple.

$1^{\circ}$. Supposons que g soit compacte. Comme nous l'avons vu dans ce qui précède le théorème 2.1 , notre assertion est bien connue dans ce cas: on a ici toujours $G_{1}=G^{0}$.

$2^{\circ}$. Supposons que $g$ soit isomorphe à la restriction scalaire d'une algèbre de Lie simple complexe $\widetilde{\mathfrak{f}}, \mathfrak{f}$ étant une forme réelle compacte de $\widetilde{\mathfrak{f}}$. Aut $(\widetilde{\mathfrak{f}})$ possède un sous-groupe fini $E^{\prime}$ tel que tout élément de $E^{\prime}$ laisse $\mathfrak{f}$ invariante

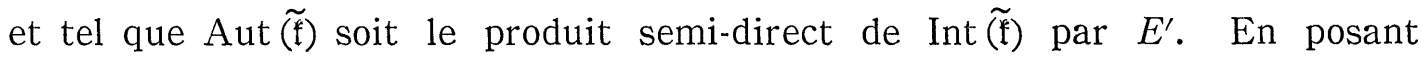
$E=E^{\prime}\{1, \theta\}$, on vérifie aussitôt notre assertion dans ce cas : on a ici toujours $G_{1}=G^{0}$.

$3^{\circ}$. Supposons que $g$ soit une forme réelle non compacte d'une algèbre de Lie simple complexe.

a. Supposons en outre que $\operatorname{Aut}(\Pi, \sigma)$ se réduise à l'élément neutre. Dans ce cas notre assertion résulte immediatement du théorème 2.1.

b. Supposons que Aut $(\Pi, \sigma)$ ne se réduise pas à l'élément neutre et que $\mathfrak{g}$ ne soit isomorphe à aucune des formes réelles normales de types $\left(D_{2 m}\right)(m \geqq 2)$. Soit alors $\xi$ un élément de $G$ vérifiant les propriétés mentionnées dans la 
proposition 2.2: l'une d'elles entraîne que $\xi$ commute à tout élément de $Q_{1}$. Posons $E=\{1, \xi\}$ et soit $S_{1}$ un sous-groupe de $Q_{1}$ tel que $Q_{1}$ soit le produit direct de $Q_{0}$ par $S_{1}$. Il est alors immédiat que le sous-groupe $S=S_{1} E$ satisfait aux conditions indiquées dans le théorème 2.2. Remarquons que $S$ se compose des éléments involutifs dans ce cas.

c. Supposons que g soit l'une des formes réelles normales de types $\left(D_{2 m}\right)$. $(m \geqq 2)$. Dans ce cas on a déjà vérifié notre assertion à la fin du numéro précédent.

La démonstration du théorème 2.2 est maintenant complète.

En résumé, quant aux algèbres de Lie simples nous avons rétabli le. résultat suivant [5]:

Proposition 2.3. Supposons g simple. Le groupe quotient $G / G^{0}$ se compose d'éléments involutifs sauf dans le cas où g soit isomorphe à l'une des suivantes: i) la restriction scalaire de l'algèbre de lie simple complexe de type $\left(\mathrm{D}_{4}\right)$, ii) la forme réelle compacte de type $\left(\mathrm{D}_{4}\right)$, iii) les formes réelles normales de types $\left(\mathrm{D}_{2 m}\right)(m \geqq 2)$. Pour celles-ci $G / G^{0}$ est isomorphe: i) à $D_{6}$, ii) à $S_{3}$, iii) à $S_{4}(m=2)$ ou à $D_{4}(m \geqq 3) ; D_{n}$ désigne ici le groupe diédrique d'ordre $2 n$ et $S_{n}$ le groupe symétrique de degré $n$.

7. Traitons finalement un cas particulier: nous supposerons à ce numéro

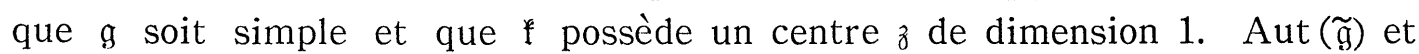
Int $(\widetilde{g})$ seront désignés, pour la raison psychologique, par $\tilde{G}_{u}$ et $\tilde{G}_{u}^{0}$ respectivement.

Soit $h$ un élément non nul dans ơ. L'endomorphisme $\operatorname{ad}(\sqrt{-1} h)$ de $\widetilde{g}$ étant semi-simple à valeurs propres réelles, soit $\tilde{\mathfrak{l}}$ la somme des sous-espaces propres correspondant aux valeurs non négatives: c'est une sous-algèbre parabolique de $\tilde{\mathfrak{g}}$. Soit $\widetilde{L}$ le sous-groupe de $\tilde{G}_{u}^{0}$ correspondant à $\tilde{\mathfrak{I}}$.

Proposition 2.4. a. Le centralisateur $K^{*}$ de z dans $K$ est d'indice 2 dans $K$, et on a $K^{*} \cap K_{1}=K^{0}$.

b. $K^{*} \widetilde{L}$ est le normalisateur $N_{\widetilde{G} u}(\widetilde{L})$ de $\widetilde{L}$ dans $\widetilde{G}_{u} . \quad N_{\widetilde{G}}(\widetilde{L}) / \widetilde{L}$ est isomorphe à $K^{*} / K^{0}$.

DÉmonstration. On note d'abord que $K^{*}$ [resp. $K^{*} \cap K_{1}$ ] est égal au centralisateur de $z$ dans $G_{u}$ [resp. $G_{u}^{0}$ ] (cf. proposition 2.1. a). Ainsi, puisque $G_{u}^{0}$ est connexe, $K^{*} \cap K_{1}$ est aussi connexe et donc égal à $K^{0}$. Soit maintenant $\mathfrak{h}_{u}$ une sous-algèbre de Cartan de $\mathfrak{g}_{u}$ contenant $\mathfrak{\jmath}$. On sait qu'il existe alors un élément $\xi \in N_{G u}\left(\mathfrak{h}_{u}\right)$ tel que $\xi \mid \mathfrak{h}_{u}=-1: \xi$ appartient à $K$ mais non à $K^{*}$. Ceci entraîne la première assertion.

On sait que $\widetilde{G}_{u}=G_{u} \widetilde{L}$ et que $\mathfrak{\mathfrak { l }}=\mathfrak{g}_{u} \cap \tilde{\mathfrak{I}}$. Par suite on a $N_{\widetilde{G}}(\widetilde{L})=N_{G_{u}}(\widetilde{L}) \widetilde{L}$, et on voit facilement que $N_{G u}(\widetilde{L})$ est identique au centralisateur de $z$ dans $G_{u}$ : d'où la deuxième assertion de la proposition 2.4.

Cela peut naturellement se traduire en termes géométriques. On sait que 
$M=G^{0} / K^{0}$ est un espace symétrique hermitien non compact irréductible et $M_{u}=G_{u}^{0} / K^{0}$ l'espace symétrique hermitien compact correspondant. $G$ est isomorphe au groupe des isométries de $M, G^{*}=K^{*} G^{0}$ au groupe $H(M)$ des transformations holomorphes de $M$, et $\widetilde{G}_{u}{ }^{*}=\widetilde{G}_{u}^{0} N_{\widetilde{G}_{u}}(\widetilde{L})$ au groupe $H\left(M_{u}\right)$ des transformations holomorphes de $M_{u}$ (cf. par exemple [3], [17]). La proposition 2.4. b signifie donc que $H(M) / H(M)^{0}$ est isomorphe à $H\left(M_{u}\right) / H\left(M_{u}\right)^{0}$ où $H(M)$ [resp. $\left.H\left(M_{u}\right)^{0}\right]$ désigne la composante connexe de l'élément neutre dans $H(M)^{0}$ [resp. $\left.H\left(M_{u}\right)\right]$.

Ajoutons enfin que la structure du groupe quotient $\tilde{G}_{u}^{*} / \tilde{G}_{u}^{0}$ peut être lue du diagramme associé à l'espace homogène kählérien $\widetilde{G}_{u}^{0} / \widetilde{L}$ : en effet le quotient est isomorphe au groupe des automorphismes du diagramme (voir [15]).

\section{$\S 3$. Sous-groupes résolubles maximaux, sous-groupes de Cartan}

8. Nous verrons à ce numéro que la question de conjugaison des sousgroupes résolubles maximaux [resp. de Cartan] d'un groupe algébrique revient à celle des sous-algèbres résolubles maximales [resp. de Cartan] d'une algèbre de Lie semi-simple.

Soient $L$ un groupe algébrique et $\mathfrak{l}$ son algèbre de Lie. Nous entendons par sous-groupe résoluble maximal [resp. de Cartan] de $L$ un sous-groupe algébrique irréductible dont l'algèbre de Lie est une sous-algèbre résoluble maximale [resp. de Cartan] de $\mathfrak{r}$.

Soient $U$ le plus grand sous-groupe unipotent distingué de $L$ et $G$ un sousgroupe réductif maximal de $L: L$ est le produit semi-direct de $U$ par $G$.

On sait que deux sous-groupes de Cartan d'un groupe algébrique résoluble sont toujours conjugués ([7]). On en déduit que tout sous-groupe de Cartan de $L$ est conjugué à celui $C$ qui contient un sous-groupe de Cartan $C_{0}$ de $G$ : $C$ est le centralisateur irréductible de $C_{0}$. Soit $C^{\prime}$ un autre sous-groupe de Carten de $L$ qui contient un sous-groupe de Cartan $C_{0}^{\prime}$ de $G$ : on voit facilement que $C$ et $C^{\prime}$ sont conjugués dans $L$ si et seulement si $C_{0}$ et $C_{0}^{\prime}$ le sont dans $G$.

Puisque $G$ est réductif, la question de conjugaison des sous-groupes de Cartan de $L$ revient ainsi à celle des sous-algèbres de Cartan de la sous-algèbre dérivée $\mathfrak{g}^{\prime}$ de $\mathfrak{g}$ sous les automorphismes (intérieurs) de $\mathfrak{g}^{\prime}$. Il en est trivialement ainsi des sous-groupes résolubles maximaux de $L$.

9. Rappelons à ce numére quelques propriétés des sous-algèbres paraboliques d'une algèbre de Lie semi-simple g. Nous reprenons les notations du $\S 2$ et les fixons une fois pour toutes dans la suite de ce paragraphe.

Un élément $x$ de $\tilde{\mathrm{g}}$ sera appelé nilpotent [resp. semi-simple, semi-simple réel] si ad $x$ est un endomorphisme nilpotent [resp. semi-simple, semi-simple à 
valeurs propres réelles]. On sait que les sous-algèbres de Cartan de g sont les sous-algèbres abéliennes maximales de g composées d'éléments semi-simples.

Soit $h$ un élément semi-simple réel de $\mathfrak{g}$ [resp. de $\widetilde{\mathfrak{g}}] . \mathfrak{g}_{0}(h), \mathfrak{g}_{+}(h)$ et $\mathfrak{g}_{*}(h)$ [resp. $\tilde{\mathfrak{g}}_{0}(h), \tilde{\mathfrak{g}}_{+}(h)$ et $\tilde{\mathfrak{g}}_{*}(h)$ ] désignerons la somme des sous-espaces de $\mathfrak{g}$ [resp. de $\tilde{\mathfrak{g}}]$ correspondant aux valeurs propres de ad $h$, respectivement, nulles, positives, et non négatives: ce sont des sous-algèbres de $\mathfrak{g}$ [resp. de $\widetilde{g}$ ].

Pour un sous-espace $\mathfrak{l}$ de $\mathfrak{g}, \mathfrak{l} \perp$ désignera le sous-espace orthogonal à $\mathfrak{l}$ par rapport à la forme de Killing de $\mathfrak{g}$.

Une sous-algèbre $\mathfrak{m}$ de $\mathfrak{g}$ est parabolique si sa complexifiée $\tilde{\mathfrak{m}}$ contient une sous-algèbre résoluble maximale de $\widetilde{\mathfrak{g}}$. On rappelle que $\tilde{\mathfrak{G}}+\sum_{\alpha>0} C e_{\alpha}$ est une sousalgèbre résoluble maximale de $\widetilde{g}$.

Proposition 3.1. Si $\mathfrak{m}$ est une sous-algèbre parabolique de $\mathfrak{g}$, on a $\mathfrak{m}=\mathfrak{g}_{*}(h)$ pour un élément $h$ semi-simple réel. Réciproquement, pour tout élément $h$ semisimple réel, $\mathrm{g}_{*}(h)$ est parabolique dans g.

DÉMONSTRATION. Soient $\mathfrak{m}$ une sous-algèbre parabolique de $\mathfrak{g}$ et $\mathfrak{c}$ une sous-algèbre de Cartan de m. Par les théorèmes de conjugaison des sous-algèbres résolubles maximales et des sous-algèbres de Cartan dans $\widetilde{\mathfrak{g}}$ et $\widetilde{\mathfrak{m}}, \tilde{\mathfrak{c}}$ est une sous-algèbre de Cartan de $\tilde{\mathfrak{g}}$. On voit sans peine que, $\tilde{\mathfrak{m}}$ étant parabolique dans $\tilde{\mathfrak{g}}$, on a $\widetilde{\mathfrak{m}}=\tilde{\mathfrak{g}}_{*}\left(h^{\prime}\right)$ pour un $h^{\prime} \in \tilde{\mathfrak{c}}$ semi-simple réel. On a alors $\widetilde{\mathfrak{m}}=\tilde{\mathfrak{g}}_{*}(h)$ aussi pour $h=\left(h^{\prime}+\sigma h^{\prime}\right) / 2 \in \mathfrak{c}$, ce qui entraîne que $\mathfrak{m}=\mathfrak{g}_{*}(h)$. La réciproque est facile à vérifier.

COROLlAIRE. Soient $\mathfrak{m}$ une sous-algèbre parabolique de $\mathrm{g}$ et $\mathfrak{n}$ son plus grand idéal composé d'éléments nilpotents. On a alors $\mathfrak{m}^{\perp}=\mathfrak{n}$.

Ceci résulte du fait que $\mathfrak{n}=\mathfrak{g}_{+}(h)$ si $\mathfrak{m}=\mathfrak{g}_{*}(h)$.

Soit $\mathcal{C}$ la chambre de Weyl positive de $\mathfrak{h}^{-}$par rapport à $\Pi^{-}$:

$$
\mathcal{C}=\left\{h \mid h \in \mathfrak{h}^{-}, \quad\left\langle\gamma_{i}, h\right\rangle \geqq 0 \quad \text { pour tout } \quad \gamma_{i} \in \Pi^{-}\right\} .
$$

Si $c$ est une sous-algèbre de Cartan de $g, c^{-}$désignera l'ensemble de ses éléments semi-simples réels, et $c^{+}$celui de ses éléments orthogonaux à $c^{-}$: on a $c=c^{+}+c^{-}$.

On démontre alors la proposition et son corollaire suivants :

Proposition 3.2. Toute sous-algèbre de Cartan de g est conjuguée par $G^{0}$ à celle c qui vérifie les conditions suivantes:

$$
\begin{aligned}
& \text { i) } \mathfrak{c}^{-} \subset \mathfrak{b}^{-}, \\
& \text {ii) } \mathfrak{c}^{+} \subset \mathfrak{f}, \\
& \text { iii) } \mathfrak{c}^{-} \cap \mathcal{C} \text { contient un élément } h \text { tel que } \mathrm{g}_{0}(h)=z\left(c^{-}\right) \text {. }
\end{aligned}
$$

COROLlaire. Toute sous-algèbre abélienne de g composée d'éléments semisimples réels est conjuguée à une sous-algèbre de $\mathfrak{h}$-. Tout élément semi-simple réel de g est conjugué à un élément de $C$. 
DÉmonstration. Soit $\mathfrak{c}$ une sous-algèbre de Cartan de g. On sait qu'il existe une forme réelle compacte $\mathfrak{g}_{u}^{*}$ de $\tilde{\mathfrak{g}}$ contenant $\mathfrak{c}^{+}+\sqrt{-1} \mathfrak{c}^{-}$et invariante par $\sigma$ (voir par exemple [12]). La proposition 3.2 et son corollaire en résultent facilement par le théorème 1.1.

Soit $h$ un élément de $\mathcal{C}$. Désignons par $\Pi(h)$ l'ensemble des racines simples s'annulant en $h$ :

$$
\Pi(h)=\left\{\alpha_{i} \mid \alpha_{i} \in \Pi,\left\langle\alpha_{i}, h\right\rangle=0\right\} .
$$

La sous-algèbre $g_{0}(h)$ est réductive, son centre étant le sous-espace orthogonal à $\Pi(h)$ dans $\mathfrak{h}$. La sous-algèbre dérivée $\mathfrak{g}_{0}(h)^{\prime}$ de $\mathfrak{g}_{0}(h)$ est invariante par $\tau$ et $\mathfrak{h}^{\prime}=\mathfrak{h} \cap \mathfrak{g}_{0}(h)^{\prime}$ est une sous-algèbre de Cartan de $g_{0}(h)^{\prime}$. En outre $\Pi(h)$ est un système $\sigma$-fondamental des racines de $\widetilde{\mathfrak{g}}_{0}(h)^{\prime}$ suivant $\widetilde{\mathfrak{h}}^{\prime}$ : le diagramme de Satake de $g_{0}(h)^{\prime}$ est donc isomorphe au sous-diagramme $(\Pi(h), \sigma)$ de $(\Pi, \sigma)$.

Pour $h$ et $h^{\prime} \in \mathcal{C}, g_{0}(h)$ et $g_{0}\left(h^{\prime}\right)$ [resp. $\mathfrak{g}_{*}(h)$ et $\mathfrak{g}_{*}\left(h^{\prime}\right)$ ] sont égales si et seulement si $\Pi(h)$ et $\Pi\left(h^{\prime}\right)$ le sont. D'autre part, pour tout sous-diagramme $\left(\Pi^{\prime}, \sigma\right)$ de $(\Pi, \sigma)$ contenant $\Pi_{0}$, il existe un $h \in \mathcal{C}$ tel que $\Pi(h)=\Pi^{\prime}$ (cf. no 1 ).

Par les propositions 3.1 et 3.2 , toute sous-algèbre parabolique de $g$ est conjuguée à l'une des $g_{*}(h)(h \in \mathcal{C})$. On sait d'ailleurs que $g_{*}(h)$ et $g_{*}\left(h^{\prime}\right)$ distincts $\left(h, h^{\prime} \in \mathcal{C}\right)$ ne sont pas conjuguées par $G^{0}$ : en effet leurs compléxifiées ne le sont pas par Int $(\widetilde{\mathfrak{g}})$ (cf. par exemple [15]). On a donc prouvé le théorème suivant:

THÉorème 3.1. Les classes de conjugaison par $G^{0}$ de sous-algèbres paraboliques de g sont en correspondance biunivoque avec les sous-diagrammes de $(\Pi, \sigma)$ contenant $\Pi_{0}$.

COROLlaire. Les classes de conjugaison par $G$ de sous-algèbres paraboliques de g sont en correspondance biunivoque avec les classes de conjugaison par Aut $(\Pi, \sigma)$ de sous-diagrammes de $(\Pi, \sigma)$ contenant $\Pi_{0}$.

10. Nous allons à ce numéro étudier les classes de conjugaison de sousalgèbres résolubles maximales et de sous-algèbres de Cartan de $\mathrm{g}$.

Proposition 3.3. Soient a une sous-algèbre résoluble maximale de $\mathfrak{g}, \mathfrak{n}$ son idéal composé des éléments nilpotents et $\mathfrak{m}$ le normalisateur de $\mathfrak{n}$ dans $\mathfrak{g}$. On a alors $\mathfrak{n}=\mathfrak{n}^{\perp}$.

DÉmonstration. Puisque $\underset{z}{ }$ est résoluble maximale dans $\mathfrak{m}, \mathfrak{n}$ se compose de tous les éléments nilpotents du radical de $\mathfrak{m}$. Par suite, $\mathfrak{m}$ étant la somme semi-directe de $\mathfrak{n}$ et d'une sous-algèbre réductive, on a $\mathfrak{m}^{\perp} \cap \mathfrak{m}=\mathfrak{n}$. Or supposons par l'absurde que $\mathfrak{n} \subsetneq \mathfrak{m}^{\perp}$. Puisqu'on a $\left[\mathfrak{n}, \mathfrak{m}^{\perp}\right] \subset \mathfrak{m}^{\perp}$, il existe alors, d'après le théorème d'Engel, un $x \in \mathfrak{m}^{\perp}$ appartenant à $\mathfrak{m}$ mais non à $\mathfrak{n}$ : ceci contredit le fait que $\mathfrak{m}^{\perp} \cap \mathfrak{m}=\mathfrak{n}$. On a donc $\mathfrak{m}^{\perp}=\mathfrak{n}$, autrement dit $\mathfrak{m}=\mathfrak{n}^{\perp}$.

COROLlaIRE 1. Si $\mathfrak{g}^{\prime}$ est une sous-algèbre semi-simple maximale de $\mathfrak{m}, \mathfrak{z} \cap \mathfrak{g}^{\prime}$ est une sous-algèbre de Cartan de $\mathfrak{g}^{\prime}$. 
DÉmonstration. $\mathfrak{g} \cap \mathfrak{g}^{\prime}$ ne possède aucun élément nilpotent non nul et donc se compose d'éléments semi-simples. Par suite $\Xi \cap g^{\prime}$ est abélienne et, étant résoluble maximale dans $\mathfrak{g}^{\prime}$, une sous-algèbre de Cartan de $\mathfrak{g}^{\prime}$.

COROLlaire 2 . $\mathfrak{m}$ est la plus petite sous-algèbre parabolique de g contenant 3 .

DÉmonstration. Soit $\mathfrak{x}$ une sous-algèbre résoluble maximale de $\widetilde{\mathfrak{g}}$ contenant $\tilde{\mathfrak{n}}$. Puisque $\tilde{\mathfrak{n}}$ se compose d'éléments nilpotents, $\tilde{\mathfrak{n}}$ est orthogonal à $\widetilde{\mathfrak{x}}: \tilde{\mathfrak{n}}^{\perp} \supset \widetilde{\mathfrak{x}}$. $\mathfrak{m}$ est donc parabolique dans $\mathfrak{g}$. Soient ensuite $\mathfrak{m}_{1}$ une sous-algèbre parabolique de $g$ contenant $g$, et $\mathfrak{n}_{1}$ son plus grand idéal composé d'éléments nilpotents. Puisque $\mathfrak{g}$ est résoluble maximale dans $\mathfrak{m}_{1}, \mathfrak{n}_{1}$ est contenue dans $\mathfrak{z}$ et même dans $\mathfrak{n}$. On a donc $\mathfrak{n}^{\perp} \subset \mathfrak{n}_{1}^{\perp}$, ce qui implique que $\mathfrak{m} \subset \mathfrak{m}_{1}$, par les propositions 3.1 et 3.3 .

Proposition 3.4. ([9]) Toute sous-algèbre résoluble maximale de g contient une sous-algèbre de Cartan de g.

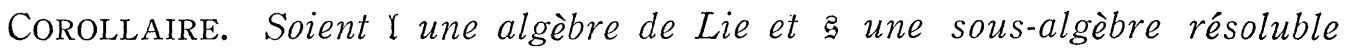
maximale de g. Z contient alors une sous-algèbre de Cartan de $\mathfrak{r}$.

DÉmonstration. Soient $\mathfrak{z}$ une sous-algèbre résoluble maximale de $\mathfrak{g}$ et $\mathfrak{m}$ la plus petite sous-algèbre parabolique de $\mathfrak{g}$ contenant $\mathfrak{g}$. Soient $\mathfrak{x}$ le radical de $\mathfrak{m}$ et $\mathfrak{g}^{\prime}$ une sous-algèbre semi-simple maximale de $\mathfrak{n}: \mathfrak{m}=\mathfrak{g}^{\prime}+\mathfrak{r}$. On a alors $\mathfrak{g}=\mathfrak{x}+\mathfrak{g} \cap \mathfrak{g}^{\prime}$, et par le corollaire 1 à la proposition $3.3, \mathfrak{g} \cap \mathfrak{g}^{\prime}$ est une sous-algèbre de Cartan de $g^{\prime}$. Il en résulte que $\mathfrak{g}$ contient une sous-algèbre de Cartan de $\mathfrak{m}$ : c'est une sous-algèbre de Cartan de $\mathfrak{g}$ puisque $\mathfrak{m}$ est parabolique dans $\mathfrak{g}$. La proposition 3.4 et son corollaire sont ainsi démontrés.

LEMme 3.1. Soit c une sous-algèbre de Cartan de g. Pour que c soit résoluble maximale dans $\mathfrak{g}$, il faut et il suffit que $\mathfrak{c}$ soit compacte.

DÉmonstration. Si c n'est pas compacte, $c$ n'est pas résoluble maximale. En effet, $c$ possède alors un élément $h$ semi-simple réel non nul et $c+g_{+}(h)$ est une algèbre résoluble strictement plus grande que $c$. Supposons ensuite que c soit compacte et soit $\xi$ une sous-algèbre résoluble maximale de $g$ contenant c. Pour vérifier que $\mathfrak{g}=\mathfrak{c}$, il suffit de montrer que $\mathfrak{g}$ ne possède aucun élément nilpotent non nul (cf. la démonstration du corollaire 1 à la proposition 3.3). Supposons par l'absurde que $\mathfrak{g}$ possède un élément nilpotent non nul et soit $\mathfrak{m}$ la plus petite sous-algèbre parabolique de g contenant 3 . Par la proposition $3.3 \mathfrak{m}$ n'est alors pas identique à g. Puisque $\mathfrak{z}$ contient le radical de $\mathfrak{m}$, par la proposition 3.1, ¿ possède un élément $h$ semi-simple réel non nul. $h$ est alors contenu dans une sous-algèbre de Cartan de $\mathcal{G}$, mais ceci contredit l'hypothèse en vertu de la conjugaison des sous-algèbres de Cartan dans $\boldsymbol{\xi}$.

LEMME 3.2. Soient $\mathfrak{m}$ une sous-algèbre parabolique de $\mathrm{g}$ et $\rightrightarrows$ une sousalgèbre résoluble maximale de $\mathrm{m}$. Z est alors résoluble maximale dans g.

DÉmonstration. Soient $\mathfrak{g}^{\prime}$ une sous-algèbre résoluble maximale de $g$ con- 
tenant $\mathfrak{g}$ et $\mathfrak{n}^{\prime}$ son idéal composé des éléments nilpotents. Puisque $\mathfrak{g}$ contient l'ensemble $\mathfrak{n}$ des éléments nilpotents du radical de $\mathfrak{m}, \mathfrak{n}^{\prime}$ aussi contient $\mathfrak{n}$. Par les propositions 3.1 et 3.3 , on a donc $\mathfrak{g}^{\prime} \subset \mathfrak{n}^{\prime \perp} \subset \mathfrak{n}^{\perp}=\mathfrak{m}$, ce qui entraîne que $\mathfrak{g}^{\prime}=\mathfrak{g}$. Le lemme 3.2 est donc démontré.

Nous pouvons maintenant prouver le théorème suivant:

THÉORÈme 3.2. En faisant correspondre une sous-algèbre résoluble maxi-

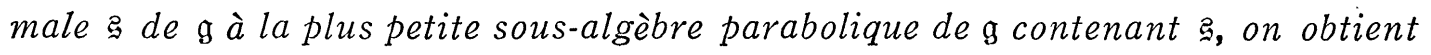
une correspondance biunivoque entre les classes de conjugaison par $G^{0}$ [resp. $G]$ de sous-algèbres résolubles maximales de g et celles de sous-algèbres paraboliques de g ayant une sous-algèbre semi-simple maximale de la première catégorie.

DÉMONSTRATION. Soient $\mathfrak{g}$ une sous-algèbre résoluble maximale de $\mathfrak{g}$ et $\mathfrak{m}$ la plus petite sous-algèbre parabolique de $g$ contenant 2 . D'après le lemme 3.1 et le corollaire 1 à la proposition $3.3, \mathfrak{m}$ possède une sous-algèbre semi-simple maximale de la première catégorie. Il est clair que la classe de conjugaison de $\mathfrak{m}$ ne dépend que de celle de 3 .

Soient maintenant $\mathfrak{n}$ une sous-algèbre parabolique de $\mathfrak{g}$ ayant une sousalgèbre semi-simple maximale $g^{\prime}$ de la première catégorie, et $\mathfrak{g}$ une sous-algèbre résoluble maximale de $\mathfrak{m}$ rencontrant $\mathfrak{g}^{\prime}$ suivant une sous-algèbre de Cartan compacte de $g^{\prime}$. D'après le lemme 3.2 g est résoluble maximale dans $g$, et, par les propositions 3.1 et $3.3, \mathfrak{m}$ est la plus petite sous-algèbre parabolique de $\mathfrak{g}$ contenant 3 . D'autre part, d'après le théorème de Levi-Malcev et celui de conjugaison des sous-algèbres de Cartan compactes, la classe de conjugaison de $\mathfrak{g}$ ne dépend que de celle de $\mathfrak{m}$. Ceci démontre que la correspondance en question est biunivoque.

COROLlaIre. Les classes de conjugaison par $G^{0}$ de sous-algèbres résolubles maximales de g sont en correspondance biunivoque avec les sous-diagrammes de $(\Pi, \sigma)$ contenant $\Pi_{0}$ et de la première catégorie.

C'est une conséquence immédiate des théorèmes 3.1 et 3.2 .

Nous étudions maintenant les classes de conjugaison de sous-algèbres de Cartan de g (cf. [8], [13]).

Notons d'abord le

LEMME 3.3. a. Soit $\mathfrak{c}$ une sous-algèbre de Cartan de g. La sous-algèbre dérivée $\left.z^{-} \mathfrak{c}^{-}\right)^{\prime}$ du centralisateur $z^{(-)}$de $\mathfrak{c}^{-}$est alors semi-simple de la première catégorie.

b. Soient $\mathfrak{c}$ et $\mathfrak{c}_{1}$ des sous-algèbres de Cartan de g. $\mathfrak{c}$ et $\mathfrak{c}_{1}$ sont conjuguées par $G^{0}$ si et seulement si $\mathrm{c}^{-}$et $\mathrm{c}_{1}^{-}$le sont.

DÉmonstration. a. $z\left(c^{-}\right)$est réductive et $c$ est une sous-algèbre de Cartan de $z\left(c^{-}\right)$. $\quad \mathfrak{c} \cap z\left(c^{-}\right)^{\prime}=c^{+} \cap z\left(c^{-}\right)^{\prime}$ est donc une sous-algèbre de Cartan de $z\left(c^{-}\right)^{\prime}$. Ceci prouve l'assertion a. 
b. Supposons que $c^{-}$et $c_{1}^{-}$soient conjuguées: $\xi\left(c_{1}^{-}\right)=c^{-}$avec $\xi \in G^{0}$. c et $\xi\left(c_{1}\right)$ sont alors des sous-algèbres de Cartan de $z\left(c^{-}\right)$. Puisque $c^{+} \cap z\left(c^{-}\right)^{\prime}$ et $\xi\left(c_{1}^{+}\right) \cap z\left(c^{-}\right)^{\prime}$ sont des sous-algèbres de Cartan compactes de $z\left(c^{-}\right)^{\prime}$, il existe un

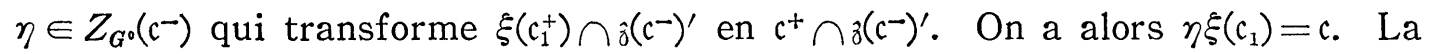
réciproque est évidente.

Soit maintenant $\mathfrak{c}$ une sous-algèbre de Cartan de $\mathfrak{g}$ vérifiant (3.2). Alors $\Pi(h)$ est l'ensemble des $\alpha_{i} \in \Pi$ s'annulant sur $c^{-}$, et donc bien déterminé par $\mathfrak{c}$ : on pose $\Pi(h)=\Pi(\mathfrak{c})$. Le centre de $z\left(\mathfrak{c}^{-}\right)$est alors le sous-espace orthogonal à $\Pi(\mathfrak{c})$ dans $\mathfrak{h}$, et $\Pi(\mathfrak{c})$ est un système $\sigma$-fondamental des racines de $\overline{3\left(\mathfrak{c}^{-}\right)^{\prime}}$ suivant $\tilde{\mathfrak{h}} \cap \overline{z\left(\mathfrak{c}^{-}\right)^{\prime}}$ (cf. no 9). D'après le lemme 3.3 le diagramme de Satake $(\Pi(\mathfrak{c}), \sigma)$ de $z\left(c^{-}\right)^{\prime}$ est de la première catégorie. Puisque $c^{-}$est le sous-espace orthogonal à $\Pi(\mathfrak{c})$ dans $\mathfrak{h}^{-}$, la dimension de $\mathfrak{c}^{-}$est égale à $p-p^{\prime}$ où $p$ [resp. $\left.p^{\prime}\right]$ est le rang restreint de $(\Pi, \sigma)$ [resp. de $(\Pi(c), \sigma)]$.

Ceci dit, on démontre la proposition suivante:

Proposition 3.5. Soient $\mathfrak{c}$ et $\mathfrak{c}_{1}$ des sous-algèbres de Cartan de g vérifiant (3.2). Pour que $\mathfrak{c}$ et $\mathfrak{c}_{1}$ soient conjuguées par $G^{0}$, il faut et il suffit qu'il existe un $s \in W_{\sigma}$ qui transforme $\Pi\left(\mathfrak{c}_{1}\right)$ en $\Pi(\mathfrak{c})$.

DÉmonstration. Supposons que $c$ et $c_{1}$ soient conjuguées par un $\xi \in G^{0}$ :

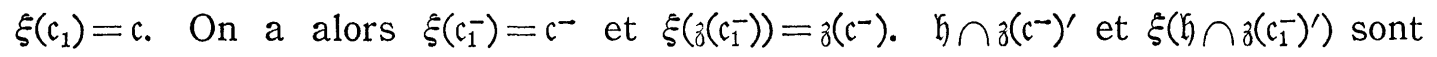
des sous-algèbres de Cartan de $z\left(c^{-}\right)^{\prime}$. Par le théorème 1.1 appliqué à $z\left(c^{-}\right)^{\prime}$, il existe un $\eta \in Z_{G^{0}}\left(\mathfrak{c}^{-}\right)$tel que $\eta \xi\left(\mathfrak{h} \cap z\left(\mathfrak{c}_{1}^{-}\right)^{\prime}\right)=\mathfrak{h} \cap \delta^{\prime}\left(\mathfrak{c}^{-}\right)^{\prime}$ et $\eta \xi\left(\Pi\left(\mathfrak{c}_{1}\right)\right)=\Pi(\mathfrak{c})$ : on a alors $\eta \xi(\mathfrak{h})=\mathfrak{h}$. On sait d'ailleurs que $N_{G^{\circ}}(\mathfrak{h})=N_{K^{\circ}}(\mathfrak{h}) \exp \mathfrak{h}$-. Par suite, si on pose $s=\eta \xi \mid \mathfrak{h}_{0}$, on a $s\left(\Pi\left(\mathfrak{c}_{1}\right)\right)=\Pi(\mathfrak{c})$ avec $s \in W_{\sigma}$. La réciproque est facile à vérifier en vertu du théorème 1.1 et du lemme 3.3.

D'après les propositions 3.2 et 3.5 , on a prouvé le théorème suivant:

THÉORÈmE 3.3. Les classes de conjugaison par $G^{0}$ [resp. G] de sousalgèbres de Cartan de $\mathfrak{g}$ sont en correspondance biunivoque avec les classes de conjugaison par $W_{\sigma}$ [resp. $T_{\sigma}$ ] de sous-diagrammes de $(\Pi, \sigma)$ contenant $\Pi_{0}$ et de la première catégorie.

Ajoutons enfin la proposition suivante [13], qui résulte facilement du théorème 3.3 et de la proposition 2.2 :

Proposition 3.6. Supposons g simple. Alors les classes de conjugaison par $G$ de sous-algèbres de Cartan de g sont identiques avec celles par $G^{0}$, sauf dans le cas où g soit isomorphe à l'une des formes réelles normales de types $\left(D_{2 m}\right)(m \geqq 2)$.

11. On verra à l'appendice le nombre de classes de conjugaison par Int (g) de sous-algèbres résolubles maximales [resp. de Cartan] pour les formes réelles non compactes $g$ des algèbres de Lie simples complexes. Nous nous bornons ici à donner un simple exemple.

Soient $V$ un espace vectoriel de dimension $n$ et $G$ le groupe des automor- 
phismes de $V$ de déterminant $1(n \geqq 0)$. Désignons par $s(n)[$ resp. $c(n)]$ le nombre de classes de conjugaison de sous-groupes résolubles maximaux [resp. de Cartan] de $G$.

Soit $D=\left(V_{0}, V_{1}, \cdots, V_{m}\right)$ un drapeau de sous-espaces vectoriels de $V$ : $V=V_{0} \supseteqq V_{1} \supseteqq \cdots \supsetneq V_{m}=(0)(m \leqq n)$. Le stabilisateur $M$ de $D$ dans $G$ est un sous-groupe parabolique de $G$, et tout sous-groupe parabolique de $G$ est le stabilisateur d'un tel drapeau convenable. Les stabilisateurs de deux drapeaux $D$ et $D^{\prime}$ sont conjugués si et seulement si $D$ et $D^{\prime}$ sont de même espèce. $M$ possède un sous-groupe semi-simple maximal de la première catégorie si et seulement si $\operatorname{dim} V_{i-1} \leqq \operatorname{dim} V_{i}+2$ pour $1 \leqq i \leqq m$. Par suite, $s(n)$ est égal au nombre de suites $\left(\nu_{1}, \nu_{2}, \cdots, \nu_{m}\right)$ composées d'entiers 1 ou 2 et ayant $n$ pour somme, et $c(n)$ à celui de répartitions de $n$ en des entiers 1 ou $2: c(n)=\left[\frac{n}{2}\right]+1$.

Remarquons que les identités suivantes sont très utiles au compte des nombres en question pour des algèbres de Lie d'autres types:

i) $s(n)+s(n+1)=s(n+2)$,

$$
\sum_{n=0}^{\infty} s(n) \lambda^{n}=\left(1-\lambda-\lambda^{2}\right)^{-1} \text {. }
$$

ii) $\sum_{i=0}^{n} s(n-i)=s(n+2)-1$,

$$
\sum_{i=0}^{\left[\frac{n}{2}\right]} s(n-2 i)=s(n+1)-\frac{1}{2}\left(1+(-1)^{n-1}\right) .
$$

iii) $c(n)=\left[\frac{n}{2}\right]+1$,

$$
\begin{aligned}
& \sum_{i=0}^{n} c(n-i)=\left(\left[\frac{n}{2}\right]+1\right)^{2}+\frac{1}{2}\left(1+(-1)^{n-1}\right)\left(\left[\frac{n}{2}\right]+1\right), \\
& \sum_{i=0}^{\left.\frac{n}{2}\right]} c(n-2 i)=\frac{1}{2}\left(\left[\frac{n}{2}\right]+1\right)\left(\left[\frac{n}{2}\right]+2\right) .
\end{aligned}
$$

\section{Appendice}

Nous donnerons une liste des résultats obtenus en suivant les méthodes exposées aux $\S \S 2$ et 3 pour les formes réelles non compactes des algèbres de Lie simples complexes.

Pour chacune d'elles, $\mathfrak{g}$, on présentera, par ordre successif, son diagramme de Satake $(\Pi, \sigma)$, éventuellement le groupe des automorphismes de celui-ci, le groupe quotient $Q_{1} / Q_{0}$ avec des relations modulo $Q_{0}$ dans $Q_{1}$, le nombre de classes de conjugaison par Int (g) de sous-algèbres résolubles maximales et de sous-algèbres de Cartan de g. Pour les notations employées, on se reportera 
aux $\S \S 2$ et 3 .

(AI) $(l \geqq 1)$

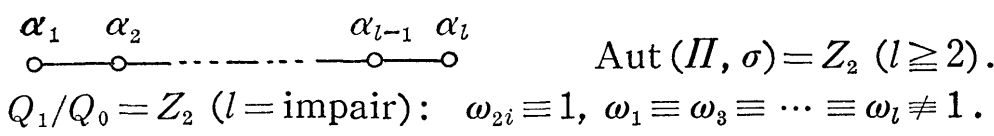

$Q_{1} / Q_{0}=\{1\}$ (l= pair).

$s(l+1)$.

$[(l+1) / 2]+1$.

(AII) $\quad(l=2 p+1 \geqq 3)$

$\stackrel{\alpha_{1}}{\circ} \cdot \ldots \stackrel{\alpha_{p}}{\bullet} \cdot \operatorname{Aut}(\Pi, \sigma)=Z_{2}$.

1 .

1 .

(AIII) $\quad(l \geqq 2,1 \leqq p \leqq l / 2)$

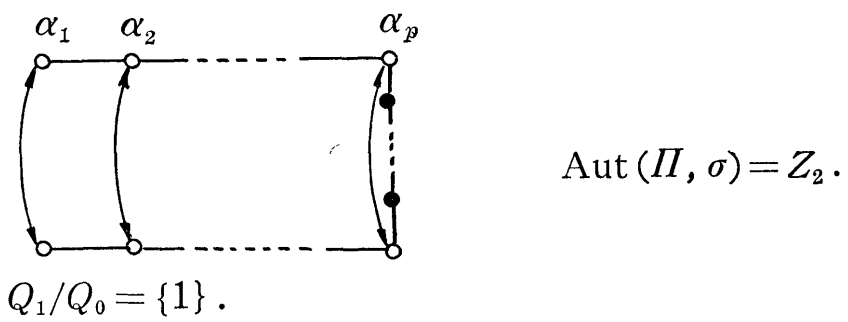

$p+1$.

$p+1$.

(AIV) $\quad(l=2 p-1 \geqq 3)$

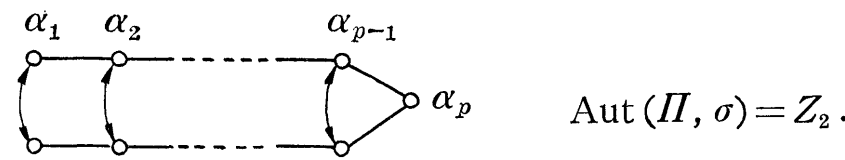

$Q_{1} / Q_{0}=Z_{2}: \omega_{1} \equiv \omega_{2} \equiv \cdots \equiv \omega_{p-1} \equiv 1, \omega_{p} \equiv 1$.

$p+1$.

$p+1$.

(BI) $(l \geqq 2,1 \leqq p \leqq l)$

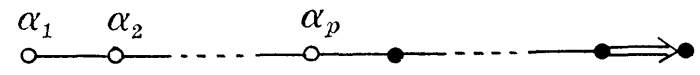

$Q_{1} / Q_{0}=Z_{2}: \quad \omega_{2 i} \equiv 1, \omega_{1} \equiv \omega_{3} \equiv \cdots \equiv \omega_{2 q-1} \equiv 1, q=\left[\frac{p+1}{2}\right]$.

$s(p+2)-1$. 
$([p / 2]+1)^{2}+\left(1+(-1)^{p-1}\right)([p / 2]+1) / 2$.

(CI) $(l \geqq 3)$

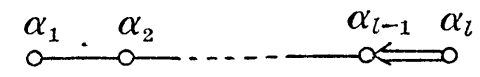

$Q_{1} / Q_{0}=Z_{2}: \omega_{1} \equiv \omega_{2} \equiv \cdots \equiv \omega_{l-1} \equiv 1, \omega_{l} \equiv 1$.

$s(l+2)-1$.

$([l / 2]+1)^{2}+1 / 2\left(1+(-1)^{l-1}\right)([l / 2]+1)$.

(CII) $\left(l \geqq 3,1 \leqq p \leqq \frac{l-1}{2}\right)$

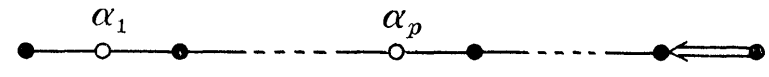

$Q_{1} / Q_{0}=\{1\}$.

$p+1$.

$p+1$.

(CIII) $\quad(l=2 p \geqq 4)$

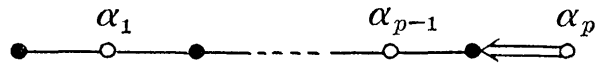

$Q_{1} / Q_{0}=Z_{2}: \omega_{1} \equiv \omega_{2} \equiv \cdots \equiv \omega_{p-1} \equiv 1, \omega_{p} \equiv 1$.

$p+1$.

$p+1$.

(DI) $(l \geqq 4,1 \leqq p \leqq l-2 ; l=p+1 \geqq 4)$

年 $\alpha_{2} \ldots$

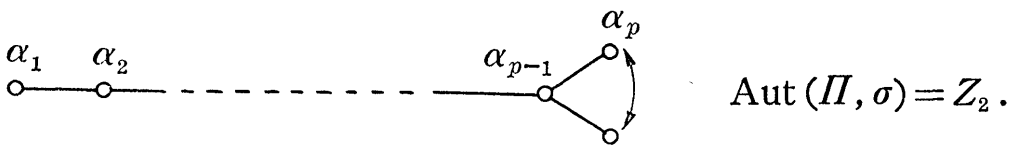

$Q_{1} / Q_{0}=\{1\}$ ( $p=$ impair).

$Q_{1} / Q_{0}=Z_{2}$ ( $p=$ pair $): \omega_{2} \equiv \omega_{4} \equiv \cdots \equiv \omega_{p} \equiv 1, \omega_{1} \equiv \omega_{3} \equiv \cdots \equiv \omega_{p-1} \equiv 1$.

$s(p+1)-\left(1+(-1)^{p-1}\right) / 2$.

$([p / 2]+1)([p / 2]+2) / 2$.

(DI) $\quad(l \geqq 4)$

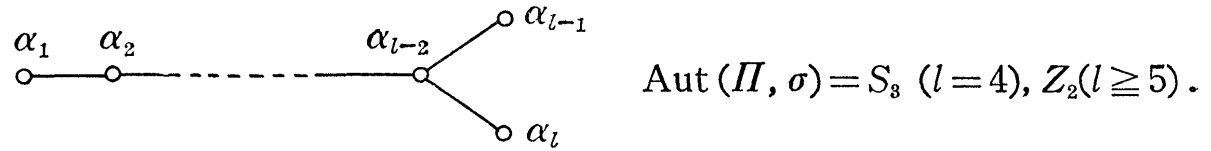


$Q_{1} / Q_{0}=Z_{2}(l=$ impair $): \omega_{1} \equiv \omega_{2} \equiv \cdots \equiv \omega_{l-2} \equiv 1, \omega_{l-1} \equiv \omega_{l} \equiv 1$.

$Q_{1} / Q_{0}=\left(Z_{2}\right)^{2}$ (l=pair): $\omega_{2} \equiv \omega_{4} \equiv \cdots \equiv \omega_{l-2} \equiv 1, \omega_{l-1} \equiv 1, \omega_{l} \equiv 1$, $\omega_{1} \equiv \omega_{3} \equiv \cdots \equiv \omega_{l-3} \equiv \omega_{l-1} \omega_{l} \equiv 1$.

$2 s(l)-\left(1+(-1)^{l-1}\right) / 2$.

$([l / 2]+1)([l / 2]+2) / 2+\left(1+(-1)^{l}\right) / 2$.

(DIII) $\quad(l \geqq 5, p=[l / 2])$

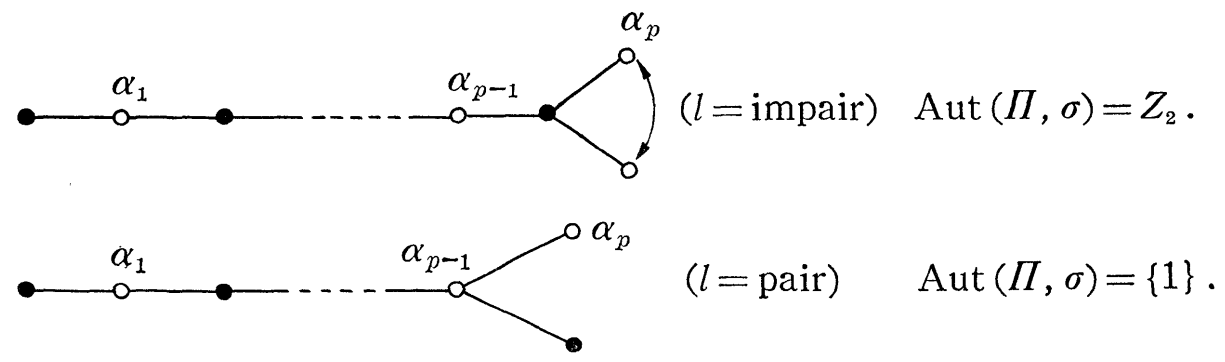

$Q_{1} / Q_{0}=\{1\}$ (l=impair).

$Q_{1} / Q_{0}=Z_{2}(l=$ pair $): \omega_{1} \equiv \omega_{2} \equiv \cdots \equiv \omega_{p-1} \equiv 1, \omega_{p} \neq 1$.

$p+1$.

$p+1$.

(EI)

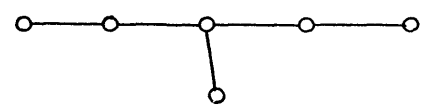

$\operatorname{Aut}(\Pi, \sigma)=Z_{2}$

$Q_{1} / Q_{0}=\{1\}$.

23 .

5 .

(EII)

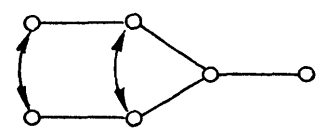

$\operatorname{Aut}(\Pi, \sigma)=Z_{2}$.

$Q_{1} / Q_{0}=\{1\}$.

6.

5 .

(EIII)

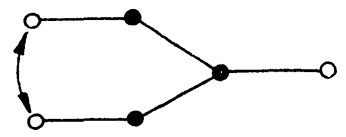

$\operatorname{Aut}(\Pi, \sigma)=Z_{2}$.

$Q_{1} / Q_{0}=\{1\}$. 


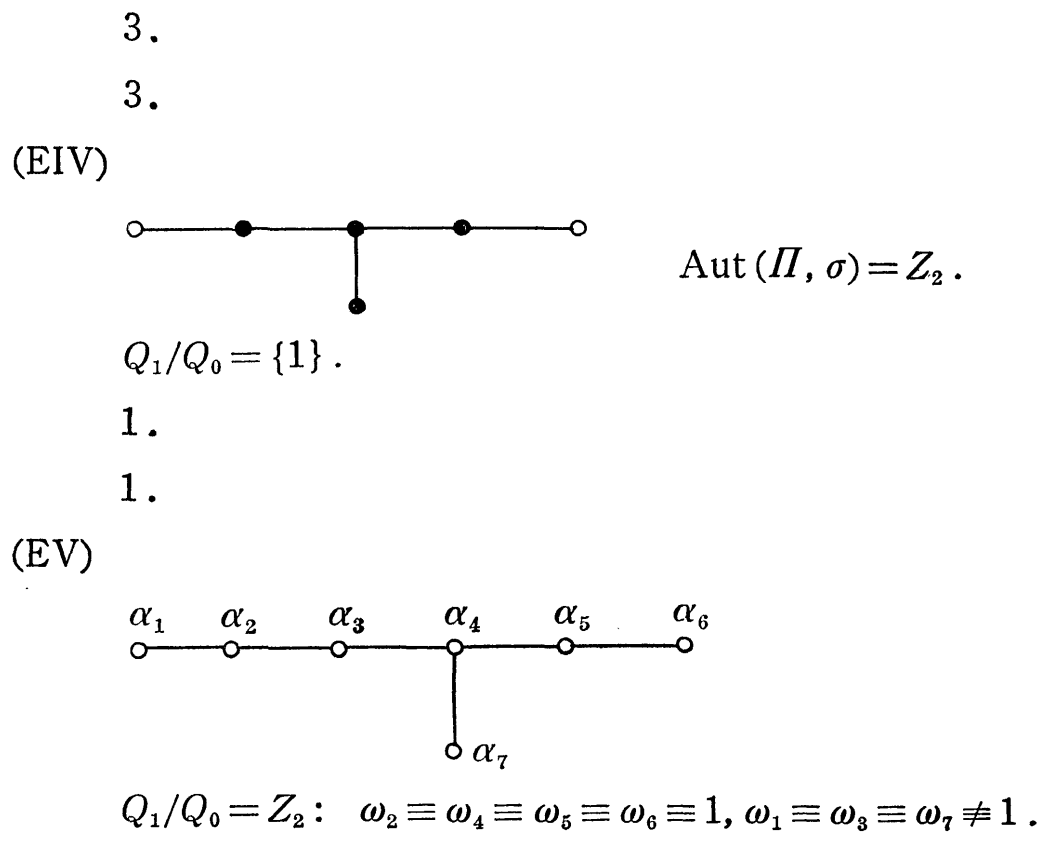

40 .

10.

(EVI)

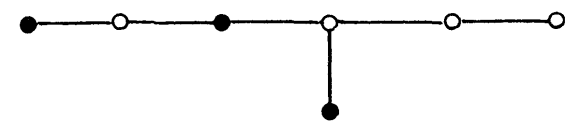

$Q_{1} / Q_{0}=\{1\}$.

6.

5.

(EVII)

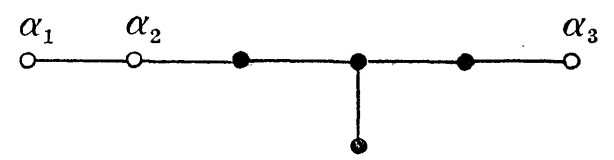

$Q_{1} / Q_{0}=Z_{2}: \omega_{2} \equiv \omega_{3} \equiv 1, \omega_{1} \equiv 1$.

4.

4.

(EVIII)

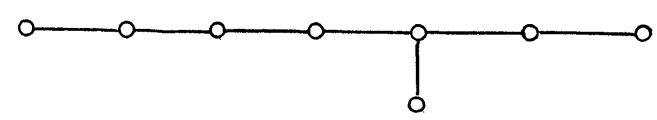

$Q_{1} / Q_{0}=\{1\}$. 
64.

10 .

(EIX)

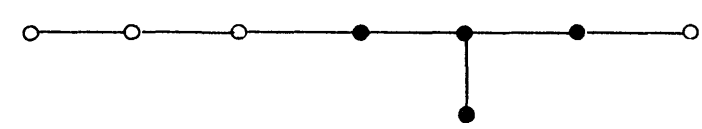

$Q_{1} / Q_{0}=\{1\}$.

6.

5 .

(FI)

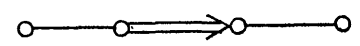

$Q_{1} / Q_{0}=\{1\}$.

12 .

8.

(FII)

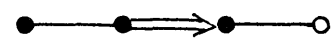

$Q_{1} / Q_{0}=\{1\}$.

2 .

2 .

(GI)

$\Longleftrightarrow 0$
$Q_{1} / Q_{0}=\{1\}$.

4 .

4 .

\author{
Institut de Mathématique \\ Université de Tokyo
}

\title{
Bibliographie
}

[1] S. Araki, On root systems and an infinitesimal classification of irreducible symmetric spaces, J. Math. Osaka City Univ., 13 (1962), 1-34.

[2] A. Borel et J. de Siebenthal, Les sous-groupes fermés de rang maximum des groupes de Lie clos, Comm. Math. Helv., 23 (1949), 200-221.

[3] A. Borel, Kählerian coset spaces of semisimple Lie groups, Proc. nat. Acad. Sc. U.S. A., 40 (1954), 1147-1151.

[4] A. Borel et Harish-Chandra, Arithmetic subgroups of algebraic groups, Ann. 
of Math., 75 (1962), 485-535.

[5] E. Cartan, Sur les formes riemanniennes remarquables des géométries à groupe fondamental simple, Ann. sc. École Norm. Sup., 44 (1927), 345-467:

[6] C. Chevalley, Théorie des groupes de Lie, II (1951), III (1955), Paris.

[7] N. Iwahori et I. Satake, On Cartan subalgebras of a Lie algebra, Kôdai math. Sem. Rep., 3 (1950), 57-60.

[8] B. Kostant, Conjugacy of real Cartan subalgebras, Proc. nat. Acad. Sc. U.S. A., 41 (1955), 967-970.

[9] G. D. Mostow, On maximal subgroups of real Lie groups, Ann. of Math., 74 (1961), 503-517.

[10] S. Murakami, On the automorphisms of a real semisimple Lie algebra, J. math. Soc. Japan, 4 (1952), 103-133; 5 (1953), 105-112.

[11] I. Satake, On representations and compactifications of symmetric Riemannian spaces, Ann. of Math., 71 (1960), 77-110.

[12] Séminaire “Sophus Lie”, 1954/1955, Théorie des algèbres de Lie, Topologie des groupes de Lie, Paris, 1955.

[13] M. Sugiura, Conjugate classes of Cartan subalgebras in real semisimple Lie algebras, J. math. Soc. Japan, 11 (1959), 374-434.

[14] M. Takeuchi, On the fundamental group and the group of isometries of a symmetric space, J. Fac. Sc. Univ. Tokyo, 10 (1964), 88-123.

[15] J. Tits, Séminaire Bourbaki, 1954/1955, exposés nos 110 et 119, Paris.

[16] J. Tits, Groupes algébriques semi-simples et géométries associées, Coll. alg. top. found. geom. (Utrecht, 1959), Oxford, 1962, 175-192.

[17] J. Tits, Espaces homogènes complexes compacts, Comm. Math. Helv., 37 (1962), 111-120. 